SPhT $92 / 073$

\title{
Polynomial Averages in the Kontsevich Model
}

\author{
P. Di Francesco, \\ C. Itzykson \\ and \\ J.-B. Zuber \\ Service de Physique Théorique de Saclay *, \\ F-91191 Gif sur Yvette Cedex, France
}

\begin{abstract}
We obtain in closed form averages of polynomials, taken over hermitian matrices with the Gaussian measure involved in the Kontsevich integral, and prove a conjecture of Witten enabling one to express analogous averages with the full (cubic potential) measure, as derivatives of the partition function with respect to traces of inverse odd powers of the external argument. The proofs are based on elementary algebraic identities involving a new set of invariant polynomials of the linear group, closely related to the general Schur functions.
\end{abstract}

$06 / 92$

* Laboratoire de la Direction des Sciences et de la Matière du Commissariat à l'Energie Atomique. 


\section{Introduction}

In their papers on intersection theory on moduli spaces of Riemann surfaces, Witten [1] and Kontsevich [2] discussed certain identities on matrix integrals. We provide here algebraic proofs for these statements which read as follows.

Let $X, Y, \Lambda \ldots$ denote $N \times N$ hermitian matrices. For $\Lambda$ positive definite, hence $\Lambda^{-1}$ well defined, introduce the measure

$$
\begin{aligned}
\mathrm{d} \mu_{\Lambda}^{(N)}(Y) & =(2 \pi)^{-N^{2} / 2} \prod_{i=1}^{N} \mathrm{~d} Y_{i i} \prod_{1 \leq i<j \leq N} \mathrm{~d} \operatorname{Re} Y_{i j} \mathrm{~d} \operatorname{I} m Y_{i j} \exp -\frac{1}{2} \operatorname{tr} \Lambda Y^{2} \\
& =\mathrm{d} Y \exp -\frac{1}{2} \operatorname{tr} \Lambda Y^{2}
\end{aligned}
$$

Proposition (K) For any polynomial $P$ in the traces of odd powers of $Y$ ("odd traces" for short), there exists a polynomial $Q$ in odd traces of $\Lambda^{-1}$, such that, independently of $N$ large enough

$$
\langle P\rangle_{(N)}\left(\Lambda^{-1}\right)=\frac{\int \mathrm{d} \mu_{\Lambda}^{(N)}(Y) P(Y)}{\int \mathrm{d} \mu_{\Lambda}^{(N)}(Y)}=Q\left(\Lambda^{-1}\right)
$$

In section 2 we exhibit more precisely the surjective map $K: P \rightarrow Q$ (proposition (K')) which turns out to be defined over $\mathbb{Q}$, the rationals, and obtain its kernel. Upon applying Wick's theorem to the computation of $\langle P\rangle$, i.e. performing a "fat graph" expansion [1][2], the proposition amounts to intricate algebraic identities, since each graph contributes a symmetric rational function of the eigenvalues of $\Lambda$. In a previous paper [3], two of the authors proved what amounts to a special case of this conjecture $(P$ was a polynomial in $\operatorname{tr} Y^{3}$ only) by a detailed but painful analysis, which admitted however a generalization to more general integrals not considered here.

It follows from $(\mathrm{K})$ that the integral

$$
\Xi_{N}\left(\Lambda^{-1}\right)=\frac{\int \mathrm{d} \mu_{\Lambda}^{(N)}(Y) \exp \frac{i}{6} \operatorname{tr} Y^{3}}{\int \mathrm{d} \mu_{\Lambda}^{(N)}(Y)}=\left\langle\exp \frac{i}{6} \operatorname{tr} Y^{3}\right\rangle_{(N)}\left(\Lambda^{-1}\right)
$$

admits as $N \rightarrow \infty$ an asymptotic expansion $\Xi(\theta$.), each term of which is a polynomial in the normalized odd traces $((-1) ! !=1)$

$$
\theta_{2 k+1}=-\frac{2}{2 k+1} \operatorname{tr} \Lambda^{-2 k-1}
$$


which become independent variables. For a more accurate definition see [3]. Similarly for $P$ a polynomial in the odd traces of $Y$, the quantity

$$
\ll P \gg_{(N)}=\left\langle P(Y) \exp \frac{i}{6} \operatorname{tr} Y^{3}\right\rangle_{(N)}\left(\Lambda^{-1}\right)
$$

admits an asymptotic expansion $\ll P \gg(\theta$. $)$, each term of which is independent of $N$ for $N$ large enough. We then have the second proposition conjectured by Witten and discussed in section 3

Proposition (W) For each $P$ as above there exists a polynomial $R$ in the derivatives $\partial_{\theta} \equiv\left\{\partial_{\theta_{1}}, \partial_{\theta_{3}}, \ldots\right\}$ such that

$$
\ll P \gg\left(\theta_{.}\right)=R\left(\partial_{\theta}\right) \Xi\left(\theta_{.}\right)
$$

The invertible mapping $P \leftrightarrow R$, defined over $\mathbb{Q}$, is given explicitely by proposition $\left(\mathrm{W}^{\prime}\right)$.

The idea underlying these proofs is simple enough as it reduces to compare calculations for matrices of size differing by a finite amount. To make this rigorous, we have to follow an indirect path which unfortunately tends to obscure the proofs in a maze of cumbersome notations. On our way we are led to introduce a set of polynomials denoted $f .(\theta$.), in infinitely many variables $\theta . \equiv\left\{\theta_{1}, \theta_{3}, . ., \theta_{2 k+1}, \ldots\right\}$, closely related to the generalized Schur

functions, in terms of which our results are most simply expressed (see eqns. (2.18) and $(2.27))$. We tabulate the first few $f$.'s as well as some expressions obtained in the text (tables I-IV).

The reader might rightly wonder about the meaning of such results. The answer is that both $(\mathrm{K})$ and $(\mathrm{W})$ enable one to construct general "observables", or topological invariants, in the combinatorial treatment of moduli spaces [1] [2].

This work was prompted by questions raised by E. Witten and M. Kontsevich [0].

\section{Proof of proposition (K)}

\subsection{Preparation}

For $X$ a generic $N \times N$ hermitian matrix with eigenvalues $x_{1}, x_{2}, \ldots, x_{N}$, and $u$ a complex variable, the rational function

$$
F_{1}(u ; X)=\operatorname{det} \frac{1-u X}{1+u X}
$$


admits for $u$ in the disk $D(X) \equiv\left\{|u|<\frac{1}{\sup _{i}\left|x_{i}\right|}\right\}$ a convergent power series

$$
F_{1}(u ; X)=\sum_{k=0}^{\infty} u^{k} p_{k}(X)
$$

Each $p_{k}$ is a polynomial in the odd traces of $X$, normalized in this section as

$$
\theta_{2 n+1}(X)=-2 \frac{\operatorname{tr} X^{2 n+1}}{2 n+1}
$$

homogeneous of degree $k$, by assigning degree $2 n+1$ to $\theta_{2 n+1}$, as follows from identifying

$$
F_{1}(u ; X)=\exp \left(-2 \sum_{n=0}^{\infty} u^{2 n+1} \operatorname{tr} \frac{X^{2 n+1}}{2 n+1}\right)=\sum_{k=0}^{\infty} u^{k} p_{k}(X)
$$

Considered as a polynomial in the infinitely many variables $\theta$. we have therefore

$$
p_{k}(\theta .)=\sum_{\substack{\nu_{j} \geq 0, j \text { odd } \\ k=\nu_{1}+3 \nu_{3}+\ldots}} \prod_{j=1,3, \ldots} \frac{\theta_{j}^{\nu_{j}}}{\nu_{j} !}
$$

By abuse of language we will call these functions Schur polynomials, although they are obtained from the standard ones by setting all the even variables $\theta_{2 j}$ to zero. We also extend the standard definition by setting $p_{k}=0$ for $k<0$.

More generally, for $k_{1}, k_{2}, \ldots, k_{n}$ non negative integers

$$
\operatorname{ch}_{k_{1}, \ldots, k_{n}}(\theta .)=\left|\begin{array}{cccc}
p_{k_{1}-n+1} & p_{k_{1}-n+2} & \ldots & p_{k_{1}} \\
p_{k_{2}-n+1} & p_{k_{2}-n+2} & \ldots & p_{k_{2}} \\
\cdot & \cdot & \ldots & \cdot \\
\cdot & \cdot & \ldots & \cdot \\
p_{k_{n}-n+1} & p_{k_{n}-n+2} & \ldots & p_{k_{n}}
\end{array}\right|(\theta .)
$$

is a polynomial in the odd traces $\theta$, antisymmetric in its indices, of degree

$$
\mathrm{d}_{\{k\}}=\sum_{r=1}^{n} k_{r}-\frac{n(n-1)}{2}
$$

which has to be non negative for $\mathrm{ch}$. to be non vanishing (observe that $\mathrm{ch}_{k_{1}, \ldots, k_{n}, 0}=$ $\left.\operatorname{ch}_{k_{1}-1, \ldots, k_{n}-1}\right)$. Again we extend the definition by requiring ch. to vanish if any of its indices is negative. Expressed in terms of $\theta$.'s, ch. is akin to an ordinary character of the linear group when we let the even $\theta$ 's vanish 1 .

1 Of course for the standard characters one sets $\theta_{n}=\operatorname{tr} \frac{X^{n}}{n}$ for any positive $n$. 
We introduce the following generating function

$$
\begin{gathered}
F_{n}\left(u_{1}, \ldots, u_{n} ; X\right)=\phi_{n}\left(u_{1}, \ldots, u_{n}\right) \prod_{1 \leq i<j \leq n} \operatorname{det} \frac{1-u_{i} X}{1+u_{i} X} \\
\phi_{n}\left(u_{1}, \ldots, u_{n}\right)=\prod_{1 \leq i<j \leq n} \frac{u_{i}-u_{j}}{u_{i}+u_{j}} \quad ; \phi_{1}=\phi_{0}=1
\end{gathered}
$$

such that

$$
\prod_{1 \leq i<j \leq n}\left(u_{i}+u_{j}\right) F_{n}\left(u_{1}, \ldots, u_{n} ; X\right)=\frac{1}{n !} \sum_{k_{1}, \ldots, k_{n}}\left|u^{k_{1}}, \ldots, u^{k_{n}}\right| \operatorname{ch}_{k_{1}, \ldots, k_{n}}(X)
$$

where the short hand notation stands for

$$
\left|u^{k_{1}}, \ldots, u^{k_{n}}\right|=\left|\begin{array}{ccc}
u_{1}^{k_{1}} & \ldots & u_{1}^{k_{n}} \\
\cdot & \ldots & \cdot \\
\cdot & \ldots & \cdot \\
u_{n}^{k_{1}} & \ldots & u_{n}^{k_{n}}
\end{array}\right|
$$

and $F_{0}=1, F_{n}=0$ for $n<0$. We can then think of $F_{n}$ as a function antisymmetric in the $n$ variables $u_{i}$ and of infinitely many variables $\theta$, by substituting for ch. in the series expansion its expression as a polynomial in the odd $\theta$.'s. The following enables us to replace in the statement of proposition $(\mathrm{K})$ any polynomial $P$ in the $\theta$.'s by a polynomial in the ch. $(\theta$.).

Lemma 1 Any homogeneous polynomial in the $\theta$.'s admits an expansion in terms of ch.'s of the same degree with ordered positive indices.

Indeed if to $k_{1}>k_{2}>\ldots>k_{n}$ we let correspond the Young tableau $T$ with $k_{1}-n+1$ boxes in the first row $k_{2}-n+2$ in the second ..., the standard Frobenius duality relation takes in our case the following form

$$
\prod_{j=0}^{\infty}\left((2 j+1) \theta_{2 j+1}\right)^{\nu_{2 j+1}}=\sum_{T ;|T|=\sum_{j}(2 j+1) \nu_{2 j+1}} \chi_{T}\left(\left[1^{\nu_{1}} 3^{\nu_{3}} \ldots\right]\right) \operatorname{ch}_{T}(\theta .)
$$

So on the one hand the definition (2.6) of ch. yields its expression in terms of $\theta$ 's through Schur polynomials, and on the other hand the above relation, where $\chi_{T}\left(\left[1^{\nu_{1}} 3^{\nu_{3}} \ldots\right]\right)$ denotes the character of the symmetric group on $|T|$ symbols evaluated on the corresponding class (involving only odd cycles), yields reciprocally an expression of any monomial in the $\theta$ 's as 
combination of ch.'s. We remark that all coefficients involved are rational since $\chi_{T}$ takes only integer values 2 .

To understand the origin of the property expressed by $(\mathrm{K})$, consider the Gaussian integral

$$
Z_{N}(\Lambda)=\int d \mu_{\Lambda}^{(N)}(Y)=\frac{1}{(\operatorname{det} \Lambda)^{1 / 2} \prod_{1 \leq i<j \leq N}\left(\lambda_{i}+\lambda_{j}\right)}
$$

where $\lambda_{1}, \ldots, \lambda_{N}$ are the positive eigenvalues of $\Lambda$ and we use the positive square root of $\operatorname{det} \Lambda$. After performing the "angular" average over the argument $Y$ [5], this becomes

$$
\begin{aligned}
\widetilde{Z}_{N}(\Lambda) & =\prod_{1 \leq i<j \leq N}\left(\lambda_{i}-\lambda_{j}\right) Z_{N}(\Lambda) \\
& =\frac{1}{(\operatorname{det} \Lambda)^{1 / 2}} \prod_{1 \leq i<j \leq N} \frac{\lambda_{i}-\lambda_{j}}{\lambda_{i}+\lambda_{j}} \\
& =\frac{(-1)^{N(N-1) / 2}}{N !} \int \prod_{i=1}^{N} \frac{d y_{i}}{\sqrt{2 \pi}} \operatorname{det}\left[e^{-\frac{\lambda_{l} y_{m}^{2}}{2}}\right]_{1 \leq l, m \leq N} \prod_{1 \leq i<j \leq N} \frac{y_{i}-y_{j}}{y_{i}+y_{j}}
\end{aligned}
$$

The integral over the eigenvalues $y_{i}$ of $Y$ is well defined since for $i \neq j$ as $y_{i}+y_{j} \rightarrow 0$ the determinant in the numerator vanishes, whereas the exponential factors (for $\Lambda>0$ ) ensure convergence at infinity. Both sides are antisymmetric in the $\lambda$ 's and up to factors we see that the (antisymmetrized) Gaussian transform of $\prod_{i<j}\left(y_{i}-y_{j}\right) /\left(y_{i}+y_{j}\right)$ is a similar expression in the $\lambda^{-1}$ 's.

This suggests to compare $Z_{N+n}$ to $Z_{N}$ with an argument of the form $\Lambda^{\prime} \oplus \Lambda$ where $\Lambda^{\prime}$ is an $n \times n$ matrix with eigenvalues $\lambda_{1}^{\prime}, \ldots, \lambda_{n}^{\prime}$ and $\Lambda$ as above. We can also split the integration variable $Y^{\prime} \oplus Y$ in the diagonal form occurring in (2.13). We integrate separately over $Y$ and $Y^{\prime}$ to get averages over functions $F_{n}$ of the argument $Y$ in terms of similar functions of $\Lambda^{-1}$. Both admit expansions in odd traces of $\Lambda^{\prime-1}$ and are at the origin of the property expressed in proposition $(\mathrm{K})$.

For analytic reasons it is however difficult to carry out this program directly. Therefore we take an indirect route based on the same idea which can be summarized as follows.

2 The lemma does not imply that the ch. $(\theta$.) are linearly independent for if $\widetilde{T}$ is the Young tableau dual to $T, \operatorname{ch}_{\widetilde{T}}(\theta$. $)=\operatorname{ch}_{T}(\theta$. $)$ as a consequence of the fact that $\chi_{T}$ and $\chi_{\widetilde{T}}$, which differ only by the signature of the permutation, are equal on classes involving only odd cycles, the latter corresponding to even permutations. Unfortunately, these are not the only relations on the $\operatorname{ch}_{T}(\theta$. $)$ which form an overcomplete system of generators. For a better choice, see below. 
We have first traded polynomials in odd traces (of $Y$ or $\Lambda^{-1}$ ) for linear combinations of $\operatorname{ch}_{T}(\theta$. $)$. In a second step we will substitute for $\operatorname{ch}_{T}(\theta$. $)$ an equivalent complete set denoted $f .(\theta$. $)$ indexed by positive integers, also antisymmetric in its indices, and shall prove the main result of this section

Proposition ( $\mathbf{K}^{\prime}$ ) Any polynomial in odd traces admits a unique expansion in terms of $f$.'s and vice versa. Moreover, independently of $N$ large enough

$$
\begin{gathered}
\left\langle f_{k_{1}, \ldots, k_{n}}\right\rangle_{(N)}\left(\Lambda^{-1}\right)=0 \text { if at least one of the } k_{i} \text { is odd } \\
\left\langle f_{2 k_{1}, \ldots, 2 k_{n}}\right\rangle_{(N)}\left(\Lambda^{-1}\right)=\prod_{s=1}^{n}\left(2 k_{s}-1\right) ! !(-1)^{k_{s}} f_{k_{1}, \ldots, k_{n}}\left(\theta .\left(\Lambda^{-1}\right)\right)
\end{gathered}
$$

We have therefore (i) to define the f's (ii) to show their equivalence with the ch's and linear independence (iii) to obtain the integrals $(2.14 a-b)$. Completing these three steps will prove the proposition.

\subsection{Definition of the $f .(\theta$.)}

For $X$ hermitian $N \times N$, let $x_{1}, \ldots, x_{N}$ be its eigenvalues assumed all distinct. We define $X_{a}$ as hermitian $N-1 \times N-1$, with eigenvalue $x_{a}$ omitted, similarly for $X_{a_{1}, a_{2}, \ldots}$.

As a meromorphic function in the variable $u_{1}^{-1}, F_{n}\left(u_{1}, \ldots, u_{n} ; X\right)$ defined in (2.8) can be expanded as a sum over its simple poles plus a contribution at infinity (arguments with a hat are to be omitted)

$$
\begin{aligned}
F_{n}\left(u_{1}, \ldots, u_{n} ; X\right) & =(-1)^{n-1} F_{n-1}\left(\hat{u}_{1}, u_{2}, \ldots, u_{n} ; X\right) \\
& -2 \sum_{a=1}^{N} \frac{u_{1} x_{a}}{1+u_{1} x_{a}} \frac{F_{n-1}\left(\hat{u}_{1}, u_{2}, \ldots, u_{n} ; X_{a}\right)}{F_{1}\left(x_{a}^{-1} ; X_{a}\right)} \\
& +2 \sum_{l=2}^{n}(-1)^{l} \frac{u_{1}}{u_{1}+u_{l}} F_{n-2}\left(\hat{u}_{1}, u_{2}, \ldots, \hat{u}_{l}, \ldots, u_{n} ; X\right)
\end{aligned}
$$

Upon iteration, this yields

$$
\begin{aligned}
F_{n}\left(u_{1}, \ldots, u_{n} ; X\right) & =\sum_{r=0}^{\infty}(-2)^{r} \sum_{1 \leq i_{1}<. .<i_{r} \leq n} \phi_{n-r}\left(u_{1}, . ., \hat{u}_{i_{1}}, . ., \hat{u}_{i_{r}}, \ldots, u_{n}\right)(-1)^{\mathcal{P}_{\{i\}}} \\
& \times \sum_{1 \leq a_{1}<\ldots, a_{r} \leq N} \frac{(-1)^{r(r-1) / 2}}{F_{r}\left(x_{a_{1}}^{-1}, \ldots, x_{a_{r}}^{-1} ; X_{a_{1}, \ldots, a_{r}}\right)} \operatorname{det}\left[\frac{u_{i_{s}} x_{a_{t}}}{1+u_{i_{s}} x_{a_{t}}}\right]_{1 \leq s, t \leq r}
\end{aligned}
$$


Here $(-1)^{\mathcal{P}_{\{i\}}}$ is the signature of the permutation $(1, \ldots, n) \rightarrow\left(i_{1}, . ., i_{r}, 1, . ., \hat{i}_{1}, . ., \hat{i}_{r}, . ., n\right)$, and $\phi$ is defined in (2.8). The first term $(r=0)$ on the right hand side is $\phi\left(u_{1}, \ldots, u_{n}\right)$ and

$$
\frac{(-1)^{r(r-1) / 2}}{F_{r}\left(x_{a_{1}}^{-1}, \ldots, x_{a_{r}}^{-1} ; X_{a_{1}, \ldots, a_{r}}\right)}=\prod_{1 \leq s<t \leq r} \frac{x_{a_{s}}+x_{a_{t}}}{x_{a_{s}}-x_{a_{t}}} \prod_{\substack{1 \leq s \leq r \\ l \in\left\{1,2, \ldots, \hat{a}_{1}, \ldots, \hat{a}_{r}, \ldots N\right\}}} \frac{x_{a_{s}}+x_{l}}{x_{a_{s}}-x_{l}}
$$

For $n, k_{i}>0$ define

$$
f_{k_{1}, \ldots, k_{n}}(X)=2^{n}(-1)^{k_{1}+\ldots+k_{n}} \sum_{1 \leq a_{1}<\ldots<a_{n} \leq N} \frac{(-1)^{n(n-1) / 2}}{F_{n}\left(x_{a_{1}}^{-1}, \ldots, x_{a_{n}}^{-1} ; X_{a_{1}, \ldots, a_{n}}\right)} \operatorname{det}\left[x_{a_{s}}^{k_{t}}\right]_{1 \leq s, t \leq n}
$$

and extend this definition to $n=0$ by setting $f(X)=1$. These functions (antisymmetric in $\left.k_{1}, \ldots, k_{n}\right)$ appear at first as rational symmetric functions of $X$. We shall soon see that they are in fact polynomials in $\theta .(X)$ and thus still well defined when some eigenvalues coincide. Let us insist on the fact that we assume all indices positive, otherwise let $f$. $=0$. We can now compare the two expansions of $\prod_{i<j}\left(u_{i}+u_{j}\right) F_{n}\left(u_{1}, \ldots, u_{n} ; X\right)$ on the polydisc $u_{i} \in D(X)$, namely

$$
\begin{aligned}
& \frac{1}{n !} \sum_{k_{1}, \ldots, k_{n} \geq 0}\left|u^{k_{1}} \ldots u^{k_{n}}\right| \operatorname{ch}_{k_{1}, \ldots, k_{n}}(\theta .(X))=\prod_{1 \leq i<j \leq n}\left(u_{i}-u_{j}\right)+\sum_{r=1}^{n} \frac{1}{r !} \times \\
& \quad \times \sum_{k_{1}, \ldots, k_{r}>0} f_{k_{1}, \ldots, k_{r}}(X) \sum_{1 \leq i_{1}<\ldots<i_{r} \leq n}(-1)^{\mathcal{P}_{\{i\}}} \prod_{1 \leq i<j \leq n}\left(u_{i}+\epsilon_{i j} u_{j}\right)\left|\begin{array}{ccc}
u_{i_{1}}^{k_{1}} & \ldots & u_{i_{1}}^{k_{r}} \\
u_{i_{1}}^{k_{1}} & \ldots & \dot{u}_{i_{r}}
\end{array}\right|
\end{aligned}
$$

where

$$
\epsilon_{i j}=\left\{\begin{aligned}
&-1 \text { if } i, j \in\left\{1, . ., \hat{i}_{1}, \ldots, \hat{i}_{r}, \ldots, n\right\} \\
& 1 \text { otherwise }
\end{aligned}\right.
$$

We need a few extra notations. Let the generalized antisymmetric Kronecker symbol be

$$
\delta_{k_{1}, \ldots, k_{r} ; m_{1}, \ldots, m_{r}}=\operatorname{det}\left[\delta_{k_{i}, m_{j}}\right]_{1 \leq i, j \leq r}
$$

and define a shift operator by

$$
g_{m} \rightarrow\left(P^{l} g\right)_{m} \equiv g_{m+l}, l \in \mathbf{Z}
$$

Finally we write $I \cup J$ for a partition of $\{1, \ldots, n\}$ into two disjoints ordered sets $I \equiv$ $\left\{i_{1}, \ldots, i_{|I|}\right\}$ and $J \equiv\left\{j_{1}, \ldots, j_{|J|}\right\},|I|+|J|=n$, and denote by $(-1)^{\mathcal{P}_{I, J}}$ the signature of the permutation $(1, \ldots, n) \rightarrow\left(i_{1}, . ., i_{|I|}, j_{1}, \ldots, j_{|J|}\right)$. 
Identifying the antisymmetric coefficient of $\left|u^{k_{1}} \ldots u^{k_{n}}\right|$ in the previous equality yields the desired linear relation between ch.'s and $f$.'s.

$$
\begin{aligned}
\operatorname{ch}_{k_{1}, \ldots, k_{n}}(\theta .(X)) & =\sum_{I \cup J}(-1)^{\mathcal{P}_{I, J}}\left\{\prod_{r<s \in I}\left(P_{k_{r}}^{-1}+P_{k_{s}}^{-1}\right) \prod_{i \in I ; j \in J}\left(P_{k_{i}}^{-1}+P_{k_{j}}^{-1}\right)\right\} \times \\
& \times f_{k_{i_{1}}, \ldots, k_{i} \mid}(X) \delta_{k_{j_{1}}, \ldots, k_{j_{|J|}} ;|J|-1,|J|-2, \ldots, 0}
\end{aligned}
$$

Explicitly for $n=1,2,3$ this reads

$$
\begin{aligned}
\operatorname{ch}_{k}(X) & =p_{k}(X)=\delta_{k, 0}+f_{k}(X) \\
\operatorname{ch}_{k_{1}, k_{2}}(X)= & \delta_{k_{1}, k_{2} ; 1,0}+f_{k_{1}-1}(X) \delta_{k_{2}, 0}+f_{k_{1}}(X) \delta_{k_{2}, 1} \\
& -f_{k_{2}-1}(X) \delta_{k_{1}, 0}-f_{k_{2}}(X) \delta_{k_{1}, 1}+f_{k_{1}-1, k_{2}}(X)+f_{k_{1}, k_{2}-1}(X) \\
\operatorname{ch}_{k_{1}, k_{2}, k_{3}}(X)= & \delta_{k_{1}, k_{2}, k_{3} ; 2,1,0} \\
+ & \sum_{\text {cycl. }}\left(f_{k_{1}-2}(X) \delta_{k_{2}, k_{3} ; 1,0}+f_{k_{1}-1}(X) \delta_{k_{2}, k_{3} ; 2,0}+f_{k_{1}}(X) \delta_{k_{2}, k_{3} ; 2,1}\right) \\
& +\sum_{\text {cycl. }}\left(\left[f_{k_{1}-1, k_{2}}(X)+f_{k_{1}, k_{2}-1}(X)\right] \delta_{k_{3}, 2}\right. \\
+ & {\left[f_{k_{1}-2, k_{2}-1}(X)+f_{k_{1}-1, k_{2}-2}(X)\right] \delta_{k_{3}, 0} } \\
+ & {\left.\left[f_{k_{1}-2, k_{2}}(X)+2 f_{k_{1}-1, k_{2}-1}(X)+f_{k_{1}, k_{2}-2}(X)\right] \delta_{k_{3}, 1}\right) } \\
+ & f_{k_{1}-2, k_{2}-1, k_{3}}(X)+f_{k_{1}-2, k_{2}, k_{3}-1}(X) \\
+ & f_{k_{1}, k_{2}-2, k_{3}-1}(X)+f_{k_{1}-1, k_{2}-2, k_{3}}(X) \\
+ & f_{k_{1}-2, k_{2}, k_{3}-1}(X)+f_{k_{1}, k_{2}-1, k_{3}-2}(X)+2 f_{k_{1}-1, k_{2}-1, k_{3}-1}(X) .
\end{aligned}
$$

While $k_{1}, k_{2}, \ldots, k_{n}$ are non negative on the l.h.s. of (2.23), we recall once more that on the right hand side any $f$. with a negative or zero index is set equal to zero 3 .

3 Eqn. 2.24 suggests a way of extending the definition of $f$.'s to zero and negative indices. For instance we could have defined $\varphi_{m}=f_{m}$ for $m \geq 1$, and $\varphi_{0}=1$ to get $\operatorname{ch}_{k}=\varphi_{k}=p_{k}$ for all $k \geq 0$. In the more interesting case with two indices, one can extend $\varphi_{k_{1}, k_{2}}=f_{k_{1}, k_{2}}$ for $k_{1}, k_{2} \geq 1$, to $\varphi_{k_{1}, 0}=f_{k_{1}}$ for $k_{1} \geq 1, \varphi_{0, k_{2}}=-f_{k_{2}}$ for $k_{2} \geq 1, \varphi_{0,0}=1$, and finally $\varphi_{-1,1}=-2$, in order to get $\operatorname{ch}_{k_{1}, k_{2}}=\varphi_{k_{1}-1, k_{2}}+\varphi_{k_{1}, k_{2}-1}$ for all $k_{1}, k_{2} \geq 0$. For a general expression for $\varphi$ see appendix A. 
We can rewrite (2.23) as follows

$$
\begin{aligned}
\operatorname{ch}_{k_{1}+n-1, k_{2}+n-2, \ldots, k_{n}}(X) & =\sum_{I \cup J}(-1)^{\mathcal{P}_{I, J}}\left\{\prod_{r<s \in I}\left(1+P_{k_{r}} P_{k_{s}}^{-1}\right) \prod_{i \in I ; j \in J}\left(1+P_{k_{i}} P_{k_{j}}^{-1}\right) \times\right. \\
& \left.\times \prod_{p<q \in J} P_{k_{p}}\right\} f_{k_{i_{1}}, \ldots, k_{i}|I|}(X) \delta_{k_{j_{1}}, \ldots, k_{j}|J|} ;|J|-1, \ldots, 0
\end{aligned}
$$

The operator $(1+Q), Q=P_{k} P_{l}^{-1}$ admits as formal inverse $(1+Q)^{-1}=\sum_{r \geq 0}(-Q)^{r}$ or $-\sum_{r \leq-1}(-Q)^{r}$. When acting on both sides of (2.25), either form yield equal finite sums as the reader will check. We can therefore invert (2.25) as

$$
\begin{aligned}
\prod_{1 \leq i<j \leq n}\left(1+P_{k_{i}} P_{k_{j}}^{-1}\right)^{-1} \operatorname{ch}_{k_{1}+n-1, \ldots, k_{n}}(X) & =\sum_{I \cup J} f_{k_{i_{1}}, \ldots, k_{i|I|}}(X) \times \\
& \times \prod_{r<s \in J} P_{k_{r}}\left(1+P_{k_{r}} P_{k_{s}}^{-1}\right)^{-1} \delta_{k_{j_{1}}, \ldots, k_{j}|J|} ;|J|-1, \ldots, 0
\end{aligned}
$$

For $k_{1}, \ldots, k_{n}>0$ the only non-vanishing contribution on the r.h.s. of (2.26) corresponds to $J=\emptyset$. Indeed when $J \neq \emptyset$, the antisymmetric Kronecker symbols always contain the constraint that at least one of the $k_{i}$ be zero or negative. Consequently, with $\mathbf{r}$ an antisymmetric $\mathbb{Z}$-valued $n \times n$ matrix, if we mean by $\mathbf{r} \geq 0$ the conditions $r_{i j} \geq 0$ for $i<j$, we find the inversion formula

$$
f_{k_{1}, \ldots, k_{n}}(X)=\sum_{\mathbf{r} \geq 0}(-1)^{\Sigma_{i<j} r_{i j}} \operatorname{ch}_{k_{1}+n-1+\Sigma_{j} r_{1 j}, k_{2}+n-2+\Sigma_{j} r_{2 j}, \ldots, k_{n}+\Sigma_{j} r_{n j}}(X)
$$

The sums over the $r$ 's on the r.h.s. are finite, since ch. $(X)$ vanishes whenever $k_{p}+n-$ $p+\sum_{j} r_{p j}<0$. We see that $f$. is indeed a polynomial in $\theta$. $(X)$ and since no reference is made to the size of the matrix $X$, the two inverse formulas (2.23) (2.26) can be thought of as relating two families of polynomials in infinitely many variables $\theta_{1}, \theta_{3}, \ldots$ Furthermore,

$$
\operatorname{deg} f_{k_{1}, \ldots, k_{n}}(\theta .)=\sum_{s=1}^{n} k_{s}
$$

we have therefore

Lemma 2 Any polynomial in $\theta_{1}, \theta_{3}, \ldots$ admits a unique expansion in terms of $f \equiv 1$, $f_{k}, k>0, f_{k_{1}, k_{2}}, k_{1}>k_{2}>0, \ldots$ 
Uniqueness is a consequence of a dimensional argument. The dimension of the vector space of polynomials of degree $n>0$ in $\theta_{1}, \theta_{3}, \ldots$ is equal to the number of partitions of $n$ in odd integers, while the dimension of the linear span of $f$.' $s$ such that $k_{1}>k_{2}>\ldots>k_{r}>0$ $\sum_{1 \leq s \leq r} k_{s}=n, r>0$, is the number of partitions of $n$ into unequal parts. The two are equal by virtue of Euler's identity

$$
\begin{aligned}
\prod_{n>0}\left(1+q^{n}\right) & =\prod_{n \geq 0} \frac{1}{\left(1-q^{2 n+1}\right)} \\
& =1+q+q^{2}+2 q^{3}+2 q^{4}+3 q^{5}+4 q^{6}+5 q^{7}+6 q^{8}+\ldots
\end{aligned}
$$

and of course all coefficients in $f$.'s are again rational.

The reader will find in appendix A some relations enabling to compute $f$.'s efficiently. We illustrate the change of basis from $f$.'s to monomials in $\theta$. in tables I and II, where we use the notation $\theta_{\left[1^{\nu_{1}} 3^{\nu_{3}} \ldots\right]}$ for the monomial $\frac{\theta_{1}^{\nu_{1}}}{\nu_{1} !} \frac{\theta_{3}^{\nu_{3}}}{\nu_{3} !} \ldots$

\subsection{Averages}

To complete the proof of (K') it is now sufficient to perform the averages $\left\langle f_{.}(\theta .(Y))\right\rangle_{(N)}$. For this we insert the original definition (2.18), taking the size of the matrices large enough (in particular $N \geq n$, the number of indices). Of course $n>0$, since for $n=0$ we have nothing to prove. Thus

$$
\begin{aligned}
\left\langle f_{k_{1}, \ldots, k_{n}}(\theta .(Y))\right\rangle_{(N)} & =\frac{(-1)^{N(N-1) / 2} 2^{n}(-1)^{k_{1}+\ldots+k_{n}}}{N ! Z_{N}(\Lambda)} \int \prod_{a=1}^{n} \frac{d y_{a}}{\sqrt{2 \pi}} \operatorname{det}\left[e^{-\frac{y_{a}^{2} \lambda_{b}}{2}}\right]_{1 \leq a, b \leq N} \times \\
& \times \prod_{\substack{1 \leq a<b \leq N\\
}} \frac{y_{a}-y_{b}}{\left(\lambda_{a}-\lambda_{b}\right)\left(y_{a}+y_{b}\right)} \sum_{1 \leq a_{1}<\ldots<a_{n} \leq N} \frac{y_{a_{r}}+y_{a_{s}}}{y_{a_{r}}-y_{a_{s}}} \times r<s \leq n \\
& \times \prod_{\substack{1 \leq t \leq n \\
l \in\left\{1, \ldots, \hat{a}_{1}, \ldots, \hat{a}_{l}, \ldots, N\right\}}} \frac{y_{a_{t}}+y_{l}}{y_{a_{t}}-y_{l}} \operatorname{det}\left[y_{a_{m}}^{k_{p}}\right]_{1 \leq m, p \leq n}
\end{aligned}
$$

We expand the $N \times N$ determinant det $e^{-\frac{y_{a}^{2} \lambda_{b}}{2}}$ according to Lagrange's formula as an alternating sum of products of determinants of size $n$ and $N-n$ respectively, take signs carefully into account and note vast cancellations, to get

$$
\begin{aligned}
& \left\langle f_{k_{1}, \ldots, k_{n}}(\theta .(Y))\right\rangle_{(N)}=\frac{(-1)^{N(N-1) / 2} 2^{n}(-1)^{k_{1}+\ldots+k_{n}}}{N ! Z_{N}(\Lambda) \prod_{1 \leq a<b \leq N}\left(\lambda_{a}-\lambda_{b}\right)} \int \prod_{a=1}^{n} \frac{d y_{a}}{\sqrt{2 \pi}} \sum_{\substack{1 \leq a_{1}<\ldots<a_{n} \leq N \\
1 \leq b_{1}<\ldots<b_{n} \leq N}} \times \\
& \prod_{\substack{a<a^{\prime} \\
a, a^{\prime} \in\left\{1, . ., \hat{a}_{1}, . ., \hat{a}_{n}, \ldots, N\right\}}}(-1)^{b_{1}+. .+b_{n}} \frac{y_{a}-y_{a^{\prime}}}{y_{a}+y_{a^{\prime}}} \operatorname{det}\left[e^{-\frac{y_{a}^{2} \lambda_{b}}{2}}\right]_{\substack{a \in\left\{1, \ldots, \hat{a}_{1}, \ldots, \hat{a}_{n}, \ldots N\right\} \\
b \in\left\{1, \ldots, \hat{b}_{1}, \ldots, \hat{b}_{n}, \ldots, N\right\}}} \times \\
& \times \operatorname{det}\left[e^{-\frac{y_{a_{s} \lambda_{b_{t}}}^{2}}{2}}\right]_{1 \leq s, t \leq n} \operatorname{det}\left[y_{a_{s}}^{k_{t}}\right]_{1 \leq s, t \leq n}
\end{aligned}
$$


For each set $1 \leq a_{1}<a_{2} . .<a_{n} \leq N$ the integral over the corresponding $y$ 's yields equal results while the integral over the remaining $y$ 's yields a factor $Z_{N-n}\left(\Lambda_{b_{1}, . ., b_{n}}\right)$

$$
\begin{aligned}
\left\langle f_{k_{1}, \ldots, k_{n}}(\theta .(Y))\right\rangle_{(N)} & =\frac{(-1)^{n(2 N-n-1) / 2} 2^{n}(-1)^{k_{1}+. .+k_{n}}}{n !} \sum_{1 \leq b_{1}<. .<b_{n} \leq N} \frac{Z_{N-n}\left(\Lambda_{b_{1}, . ., b_{n}}\right)}{Z_{N}(\Lambda)} \times \\
& \times(-1)^{b_{1}+. .+b_{n}} \frac{\prod_{b<b^{\prime} \in\left\{1, . ., \hat{b}_{1}, . ., \hat{b}_{n}, . ., N\right\}}\left(\lambda_{b}-\lambda_{b^{\prime}}\right)}{\prod_{1 \leq b<b^{\prime} \leq n}\left(\lambda_{b}-\lambda_{b^{\prime}}\right)} \times \\
& \times \int \prod_{t=1}^{n} \frac{d y_{t}}{\sqrt{2 \pi}} \operatorname{det}\left[e^{-\frac{y_{s}^{2} b_{t}}{2}}\right]_{1 \leq s, t \leq n} \operatorname{det}\left[y_{s}^{k_{t}}\right]_{1 \leq s, t \leq n}
\end{aligned}
$$

The last integral vanishes whenever one of the $k_{i}$ at least is odd, hence we get $\left.2.14 a\right)$

$$
\left\langle f_{k_{1}, \ldots, k_{n}}(\theta .(Y))\right\rangle_{(N)}=0 \text { if at least one } k_{i} \neq 0 \bmod 2
$$

whereas using $\int d y y^{2 k} e^{-\lambda y^{2} / 2}=\sqrt{2 \pi / \lambda}(2 k-1) ! ! \lambda^{-k}$, we get

$$
\begin{aligned}
& \left\langle f_{2 k_{1}, \ldots, 2 k_{n}}(\theta .(Y))\right\rangle_{(N)}=2^{n}(-1)^{n(2 N-n-1) / 2} \prod_{s=1}^{n}\left(2 k_{s}-1\right) ! ! \times \\
& \times \sum_{1 \leq b_{1}<. .<b_{n} \leq N} \prod_{1 \leq s<t \leq n} \frac{\lambda_{b_{s}}+\lambda_{b_{t}}}{\lambda_{b_{s}}-\lambda_{b_{t}}} \prod_{\substack{1 \leq s \leq n \\
b \in\left\{1, \ldots, \hat{b}_{1}, \ldots, \hat{b}_{n}, \ldots, N\right\}}} \frac{\lambda_{b_{s}}+\lambda_{b}}{\lambda_{b_{s}}-\lambda_{b}} \operatorname{det}\left[\lambda_{b_{t}}^{k_{s}}\right]_{1 \leq s, t \leq n}
\end{aligned}
$$

Since $\left(\lambda_{b}+\lambda_{b^{\prime}}\right) /\left(\lambda_{b}-\lambda_{b^{\prime}}\right)=-\left(\lambda_{b}^{-1}+\lambda_{b^{\prime}}^{-1}\right) /\left(\lambda_{b}^{-1}-\lambda_{b^{\prime}}^{-1}\right)$, comparing with the definition of $f .\left(\theta .\left(\Lambda^{-1}\right)\right)$, we obtain $(2.14 b)$ in the form

$$
\left\langle f_{2 k_{1}, \ldots, 2 k_{n}}(\theta .(Y))\right\rangle_{(N)}=(-1)^{k_{1}+. .+k_{n}} \prod_{s=1}^{n}\left(2 k_{s}-1\right) ! ! f_{k_{1}, \ldots, k_{n}}\left(\theta .\left(\Lambda^{-1}\right)\right)
$$

as claimed. This completes the proof of proposition $\left(\mathrm{K}^{\prime}\right)$. We add a few comments.

(i) As shown along the way the map $K: P\left(\theta_{\text {. }}\right) \rightarrow Q\left(\theta_{\text {. }}\right)$ is defined over $\mathbb{Q}$.

(ii) Since $f$.'s generate all polynomials in odd traces, this map is surjective. Its kernel is the linear span

$\operatorname{Ker}(K)=\left\{\right.$ linear span of $f_{k_{1}, \ldots, k_{n}}(\theta),. n>0$, such that at least one of the $k_{i}$ is odd $\}$ Indeed any $P(\theta$. $)$ is a finite sum

$$
P(\theta .)=a_{0}+\sum_{n>0} \sum_{k_{1}>k_{2}>\ldots>k_{n}>0} a_{k_{1}, . ., k_{n}} f_{k_{1}, \ldots, k_{n}}(\theta .)
$$


if $K(P)=0$, it follows that

$$
\sum_{k_{1}>\ldots>k_{n}>0} a_{2 k_{1}, \ldots, 2 k_{n}} f_{k_{1}, . ., k_{n}}(\theta .)=0 \Rightarrow a_{2 k_{1}, . ., 2 k_{n}}=0
$$

Therefore if $d(n)$ denotes the dimension of the vector space of polynomials of degree $n$ in $\theta$.'s and $d_{0}(n)$ the dimension of the subspace annihilated by $K$, we have

$$
\begin{aligned}
\sum_{n=0}^{\infty} d(n) q^{n} & =\prod_{n>0}\left(1+q^{n}\right) \\
\sum_{n=0}^{\infty} d_{0}(n) q^{n} & =\prod_{n>0}\left(1+q^{n}\right)-\prod_{n>0}\left(1+q^{2 n}\right) \\
& =\prod_{n \geq 0} \frac{1}{\left(1-q^{2 n+1}\right)}\left(1-\prod_{n \geq 0} \frac{1}{\left(1+q^{2 n+1}\right)}\right) \\
& =q+2 q^{3}+q^{4}+3 q^{5}+2 q^{6}+5 q^{7}+4 q^{8}+\ldots
\end{aligned}
$$

\section{Proof of Proposition (W)}

Notations being as before, we consider the integral (1.3)

$$
\Xi_{N}\left(\Lambda^{-1}\right)=\frac{\int \mathrm{d} \mu_{\Lambda}^{(N)}(Y) \exp \frac{i}{6} \operatorname{tr} Y^{3}}{\int \mathrm{d} \mu_{\Lambda}^{(N)}(Y)}=\left\langle\exp \frac{i}{6} \operatorname{tr} Y^{3}\right\rangle_{(N)}\left(\Lambda^{-1}\right)
$$

which admits an asymptotic expansion, each term of which is for $N$ sufficiently large an $N$-independent polynomial in the odd traces of $\Lambda^{-1}$. We keep the normalization (2.3)

$$
\theta_{2 k+1}\left(\Lambda^{-1}\right)=-\frac{2}{2 k+1} \operatorname{Tr} \Lambda^{-2 n-1}
$$

As $N$ tends to infinity these become independent variables and the asymptotic expansion is denoted $\Xi(\theta$. $)$. For any polynomial in odd traces $P(Y) \equiv P\left(\operatorname{tr} Y, \operatorname{tr} Y^{3}, \ldots\right)$ set

$$
\ll P \gg_{N}=\left\langle P(Y) \exp \left(\frac{i}{6} \operatorname{tr} Y^{3}\right\rangle_{N}\left(\Lambda^{-1}\right)\right.
$$

From section 2 it admits an $N$-independent asymptotic expansion $\ll P \gg(\theta$.) in the odd traces $\theta_{2 k+1}\left(\Lambda^{-1}\right)$. Explicit calculations performed by Witten [1] suggest that one can express this average as

$$
\ll P \gg=R\left(\frac{\partial}{\partial \theta}\right) \Xi(\theta .)
$$


where $R$ is again a polynomial (with constant coefficients) in the derivatives $\frac{\partial}{\partial \theta_{2 k+1}}$, and conversely that for any such $R$ there exists a $P$. Using techniques developed in [3], one can for instance derive closed expressions for $R$ a monomial in $\theta_{1}$ or $\theta_{3}$ (see appendix B)

$$
\begin{aligned}
& \left(\frac{\partial}{\partial \theta_{1}}\right)^{k} \Xi(\theta .)=\sum_{\substack{m, n \geq 0 \\
3 n+m=k}} \frac{(3 n+m) !}{6^{n} n ! m !} \ll\left(\operatorname{tr} \frac{Y}{2 i}\right)^{m} \gg \\
& \left(\frac{\partial}{\partial \theta_{3}}\right)^{k} \Xi(\theta .)=\left.\left((1+3 y / 4)^{3} \partial_{y}\right)^{k}(1+3 y / 4)^{\frac{1}{12}} \ll e^{y \operatorname{tr}\left(\frac{Y}{2 i}\right)^{3}} \gg\right|_{y=0}
\end{aligned}
$$

and a more cumbersome, although perfectly explicit formula for $R$ being any polynomial in both $\partial_{\theta_{1}}$ and $\partial_{\theta_{2}}$ (see appendix $\mathrm{B}$ for details).

The proof of proposition (W) is based as before on the comparison of the integral over $N \times N$ matrices $\Xi_{N}$, defined in (3.1), to the same integral $\Xi_{N+n}$ over $(N+n) \times(N+n)$ matrices. Indeed it is easy to see for $n=1$ that in the expansion of $\Xi_{N+1}\left(\lambda^{-1} \oplus \Lambda^{-1}\right)$, where $\lambda$ is a real positive number and $\Lambda$ a positive definite diagonal $N \times N$ matrix, the terms of degree $3 k \leq N$ are obtained from those of the same degree in the expansion of $\Xi_{N}\left(\Lambda^{-1}\right)$ by translating the variables $\theta .\left(\Lambda^{-1}\right)$ according to

$$
\theta_{2 j+1}\left(\Lambda^{-1}\right) \rightarrow \theta_{2 j+1}\left(\lambda^{-1} \oplus \Lambda^{-1}\right)=\theta_{2 j+1}\left(\Lambda^{-1}\right)-\frac{2}{2 j+1} \lambda^{-2 j-1}
$$

Therefore in the usual $N \rightarrow \infty$ limit, we can write

$$
\begin{aligned}
\Xi\left(\lambda^{-1} \oplus \Lambda^{-1}\right) & =\exp \sum_{i=0}^{\infty}-\frac{2}{2 j+1} \lambda^{-2 j-1} \frac{\partial}{\partial \theta_{2 j+1}} \Xi\left(\Lambda^{-1}\right) \\
& =\sum_{k=0}^{\infty} \lambda^{-k} p_{k}\left(\partial_{.}\right) \Xi
\end{aligned}
$$

We see that the power series in $\lambda^{-1}$ on the r.h.s. is a generating function for the Schur polynomials (defined in (2.5)) of derivatives $\partial$.

$$
\begin{aligned}
\partial_{2 j+1} & \equiv-\frac{2}{2 j+1} \frac{\partial}{\partial \theta_{2 j+1}} \\
\partial_{2 j} & \equiv 0
\end{aligned}
$$

acting on $\Xi$, as a function of the infinitely many variables $\theta_{2 j+1}$. Increasing $n$ amounts to iterating this process, and we get in general a generating function for any product of Schur polynomials of $\partial$. acting on $\Xi$. These products span the whole space of polynomials in the variables $\partial_{2 j+1}$. 
We are left with the task of computing the l.h.s. of (3.7) and its generalizations. Proposition (W) will follow if we can find expressions of the former as generating functions for expectation values of polynomials of the form (1.5). This last step turns out to be elementary, and leads to explicit expressions for the aforementioned polynomials. It is summarized in the following

Lemma 3 Let $\Lambda=\Lambda_{1} \oplus \Lambda_{2}$ be the decomposition of the diagonal matrix $\Lambda=$ $\operatorname{diag}\left(\lambda_{1}, \ldots, \lambda_{N+n}\right)$ into the direct sum of two diagonal matrices $\Lambda_{1}=\operatorname{diag}\left(\lambda_{1}, \ldots, \lambda_{n}\right)$ and $\Lambda_{2}=\operatorname{diag}\left(\lambda_{n+1}, . ., \lambda_{n+N}\right)$. We have

$$
\begin{aligned}
\prod_{1 \leq i<j \leq n} \frac{\lambda_{i}-\lambda_{j}}{\lambda_{i}+\lambda_{j}} \Xi_{n+N}\left(\Lambda_{1}^{-1} \oplus \Lambda_{2}^{-1}\right)=\int \prod_{k=1}^{n} d \nu_{\lambda_{k}}\left(y_{k}\right) \times \\
\prod_{1 \leq m<p \leq n} \frac{2 i\left(\lambda_{m}-\lambda_{p}\right)+y_{m}-y_{p}}{2 i\left(\lambda_{m}+\lambda_{p}\right)+y_{m}+y_{p}} \ll \prod_{l=1}^{n} \operatorname{det}\left(\frac{2 i \lambda_{l}+y_{l}-Y_{2}}{2 i \lambda_{l}+y_{l}+Y_{2}}\right) \gg_{N}\left(\Lambda_{2}^{-1}\right)
\end{aligned}
$$

where $d \nu_{\lambda}(y)=(\lambda / 2 \pi)^{\frac{1}{2}} \exp \left(i y^{3} / 6-\lambda y^{2} / 2\right) d y$ is the measure of integration over the eigenvalues $y$ adapted to our problem, and the double bracket denotes the integral over the $N \times N$ matrix $Y_{2}$ as defined in (3.3).

The lemma will be used to expand both sides of (3.9), when $N \rightarrow \infty$, as formal series in $\lambda_{j}^{-1}, 1 \leq j \leq n$, for large $\lambda^{\prime}$ s. On the l.h.s. of (3.9) we get as coefficients of this series polynomials in the derivatives w.r.t. $\theta .\left(\Lambda_{2}^{-1}\right)$, whereas on the r.h.s. one gets averages of polynomials in the odd traces of $Y_{2}$, which completes the proof of proposition $(\mathrm{W})$. Before proving lemma 3, let us illustrate the mechanism in the case $n=1$, where (3.9) reduces to

$$
\Xi_{N+1}\left(\lambda^{-1} \oplus \Lambda^{-1}\right)=\int d \nu_{\lambda}(y) \ll \operatorname{det}\left(\frac{y+2 i \lambda-Y}{y+2 i \lambda+Y}\right) \gg_{N}
$$

Expanding both sides in powers of $\lambda^{-1}$, using

$$
\Xi_{N+1}\left(\lambda^{-1} \oplus \Lambda^{-1}\right)=\int d \nu_{\lambda}(y) \ll \sum_{m \geq 0}\left(\lambda-\frac{i y}{2}\right)^{-m} p_{m}\left(\theta \cdot\left(\frac{Y}{2 i}\right)\right) \gg_{N}
$$

with $N \rightarrow \infty$, and integrating term by term over $y$, we can identify the coefficient of $\lambda^{-k}$ in $(3.7)$ as

$$
p_{k}\left(\partial_{.}\right) \Xi(\theta .)=\sum_{0 \leq s \leq[k / 3]}(-1)^{s} c_{s, k} \ll p_{k-3 s}\left(\theta .\left(\frac{Y}{2 i}\right)\right) \gg_{N}
$$


where

$$
c_{s, k}=\sum_{l=0}^{2 s} \frac{1}{2^{l}} \frac{(k-3 s+l-1) !}{l !(k-3 s-1) !} \frac{(6 s-2 l-1) ! !}{6^{2 s-l}(2 s-l) !}
$$

and $[x]$ denotes the integral part of $x$. For $k=0$, (3.12) reduces to the identity $\ll 1 \gg=$ $\Xi(\theta$.). The general case with $n>1$ will be dealt with below. Let us turn to the proof of lemma 3.

At first the matrices $\Lambda, \Lambda_{1}, \Lambda_{2}$ involve diagonal real positive elements, but if we introduce a cut in the complex plane along the negative real axis, the integrals make sense for each eigenvalue having a positive real part - as absolutely convergent integrals; as semi-convergent ones we can even extend them to the imaginary axis except the origin. To give a meaning to the following operations we will first continue analytically the $\lambda_{j}$ to imaginary non vanishing values. Similar techniques were implicit in both [2] and [3]. With this proviso in mind we return to

$$
\Xi_{n+N}\left(\Lambda^{-1}\right)=\frac{1}{Z_{n+N}(\Lambda)} \int d Y e^{\frac{i}{6} \operatorname{tr}\left(Y^{3}\right)-\frac{1}{2} \operatorname{tr}\left(\Lambda Y^{2}\right)}
$$

where $Z_{n+N}(\Lambda)$ is defined in (2.12). We perform the change of variables $Z=Y+2 i\left(\Lambda_{1} \oplus 0\right)$, with the obvious definition for the $(n+N) \times(n+N)$ matrix $\Lambda_{1} \oplus 0=\operatorname{diag}\left(\lambda_{1}, . ., \lambda_{n}, 0, . ., 0\right)$. Due to the relation

$$
\left(\Lambda_{1} \oplus 0\right)\left(0 \oplus \Lambda_{2}\right)=\left(0 \oplus \Lambda_{2}\right)\left(\Lambda_{1} \oplus 0\right)=0
$$

the trace in the exponential becomes

$$
\frac{i}{6} \operatorname{tr}\left(Y^{3}\right)-\frac{1}{2} \operatorname{tr}\left(\Lambda Y^{2}\right)=\frac{i}{6} \operatorname{tr}\left(Z^{3}\right)-\frac{1}{2} \operatorname{tr}\left(\left[\left(0 \oplus \Lambda_{2}\right)-\left(\Lambda_{1} \oplus 0\right)\right] Z^{2}\right)+\frac{2}{3} \operatorname{tr}\left(\Lambda_{1}^{3}\right)
$$

We see that except for a constant term, the form of the exponential term is conserved, up to the substitution $\Lambda=\Lambda_{1} \oplus \Lambda_{2} \rightarrow \tilde{\Lambda}=\left(0 \oplus \Lambda_{2}\right)-\left(\Lambda_{1} \oplus 0\right)$. We can now perform the "angular" average over $Z$ [5], which results in

$$
\Xi_{n+N}\left(\Lambda^{-1}\right)=\prod_{1 \leq i<j \leq N+n} \frac{\lambda_{j}+\lambda_{i}}{\tilde{\lambda_{j}}-\tilde{\lambda_{i}}} \int \prod_{k=1}^{n+N} d \nu_{\tilde{\lambda}_{k}}\left(z_{k}\right) \prod_{p=1}^{n} e^{\frac{2}{3} \lambda_{p}^{3}} \prod_{1 \leq l<m \leq N+n} \frac{z_{l}-z_{m}}{z_{l}+z_{m}}
$$

where the $\tilde{\lambda}$ 's are the diagonal elements of $\tilde{\Lambda}$, i.e. $\tilde{\lambda_{k}}=-\lambda_{k}$ for $1 \leq k \leq n, \tilde{\lambda_{k}}=\lambda_{k}$ for $n+1 \leq k \leq n+N$ (recall that the $\tilde{\lambda}$ 's are purely imaginary, so that the minus sign causes no harm in the integral). As usual the antisymmetry of the integrand in $z$ 's in (3.16) automatically takes care of the denominators $z_{l}+z_{m}$, by antisymmetrizing the measure. 
We now perform the opposite change of variables, but this time on the eigenvalues $z$ by setting

$$
\begin{aligned}
& z_{k}=y_{k}+2 i \lambda_{k} \quad 1 \leq k \leq n \\
& z_{k}=y_{k} \quad n+1 \leq k \leq n+N
\end{aligned}
$$

which leads to

$$
\begin{aligned}
& \Xi_{n+N}\left(\Lambda_{1}^{-1} \oplus \Lambda_{2}^{-1}\right)=\prod_{1 \leq i<j \leq n} \frac{\lambda_{i}+\lambda_{j}}{\lambda_{i}-\lambda_{j}} \prod_{n+1 \leq l<m \leq n+N} \frac{\lambda_{l}^{-1}+\lambda_{m}^{-1}}{\lambda_{l}^{-1}-\lambda_{m}^{-1}} \prod^{N+n} d \nu_{\lambda_{k}}\left(y_{k}\right) \times \\
& \times \prod_{1 \leq l<m \leq n} \frac{y_{l}-y_{m}+2 i\left(\lambda_{l}-\lambda_{m}\right)}{y_{l}+y_{m}+2 i\left(\lambda_{l}+\lambda_{m}\right)} \prod_{n+1 \leq i<j \leq n+N} \frac{y_{i}-y_{j}}{y_{i}+y_{j}} \prod_{\substack{1 \leq l \leq n \\
n+1 \leq j \leq n+N}} \frac{y_{l}+2 i \lambda_{l}-y_{j}}{y_{l}+2 i \lambda_{l}+y_{j}}
\end{aligned}
$$

and amounts to the statement of lemma 3 since

$$
\begin{aligned}
& \prod_{n+1 \leq l<m \leq n+N} \frac{\lambda_{l}^{-1}+\lambda_{m}^{-1}}{\lambda_{l}^{-1}-\lambda_{m}^{-1}} \int \prod_{k=n+1}^{n+N} d \nu_{\lambda_{k}}\left(y_{k}\right) \prod_{n+1 \leq i<j<n+N} \frac{y_{i}-y_{j}}{y_{i}+y_{j}} \times \\
& \times \prod_{\substack{1 \leq l \leq n \\
n+1 \leq j \leq n+N}} \frac{y_{l}+2 i \lambda_{l}-y_{j}}{y_{l}+2 i \lambda_{l}+y_{j}}=\ll \prod_{1 \leq l \leq n} \operatorname{det}\left(\frac{y_{l}+2 i \lambda_{l}-Y_{2}}{y_{l}+2 i \lambda_{l}+Y_{2}}\right) \gg_{N}\left(\Lambda_{2}^{-1}\right)
\end{aligned}
$$

\section{Remarks}

(i) Once we are through with the proof, the lemma remains valid as a statement on asymptotic series for real positive eigenvalues $\lambda$ 's.

(ii) The lemma enables us to give several expressions for the same $\Xi_{N}$ by splitting the $N$ eigenvalues of $\Lambda$ into two sets $N=N_{1}+N_{2}$. For instance we find for $N=2$ the various expressions with $\Lambda=\operatorname{diag}\left(\lambda_{1}, \lambda_{2}\right)$

$$
\begin{aligned}
\Xi_{2}\left(\Lambda^{-1}\right) & =\int \prod_{k=1,2} d \nu_{\lambda_{k}}\left(y_{k}\right) \frac{y_{1}-y_{2}+2 i\left(\lambda_{1}-\lambda_{2}\right)}{y_{1}+y_{2}+2 i\left(\lambda_{1}+\lambda_{2}\right)} \times \frac{\lambda_{1}+\lambda_{2}}{\lambda_{1}-\lambda_{2}} \\
& =\int \prod_{k=1,2} d \nu_{\lambda_{k}}\left(y_{k}\right) \frac{y_{1}+2 i \lambda_{1}-y_{2}}{y_{1}+2 i \lambda_{1}+y_{2}} \\
& =\int \prod_{k=1,2} d \nu_{\lambda_{k}}\left(y_{k}\right) \frac{y_{1}-y_{2}}{y_{1}+y_{2}} \times \frac{\lambda_{1}+\lambda_{2}}{\lambda_{2}-\lambda_{1}}
\end{aligned}
$$

where ( $3.20 \mathrm{~B})$ admits an alternative expression with $\lambda_{1} \leftrightarrow \lambda_{2}$ and (3.20d) is understood after antisymmetrization of the measure. It is interesting to check that the difference of any two of these expressions can be written as the integral of a total derivative (and therefore vanishes)! 
With lemma 3 in hand, we can now obtain the most general combination of derivatives acting on $\Xi\left(\theta\right.$.). The l.h.s. of (3.9) for $N \rightarrow \infty$ converges as an asymptotic series in $\theta$. $\left(\Lambda^{-1}\right)$ to

$$
\begin{aligned}
\prod_{1 \leq i<j \leq n} \frac{\lambda_{i}-\lambda_{j}}{\lambda_{i}+\lambda_{j}} & \Xi\left(\lambda_{1}^{-1} \oplus . . \oplus \lambda_{n}^{-1} \oplus \Lambda^{-1}\right) \\
& =\prod_{1 \leq i<j \leq n} \frac{\lambda_{i}-\lambda_{j}}{\lambda_{i}+\lambda_{j}} \sum_{r_{1}, r_{2}, . ., r_{n}} \prod_{i=1}^{n} \lambda_{i}^{-r_{i}} p_{r_{1}}\left(\partial_{.}\right) \ldots p_{r_{n}}\left(\partial_{.}\right) \Xi\left(\theta .\left(\Lambda^{-1}\right)\right)
\end{aligned}
$$

Expanding the prefactor as a power series in the domain $\lambda_{1}^{-1}>\lambda_{2}^{-1}>\ldots>\lambda_{n}^{-1}$ and performing a similar expansion on the r.h.s. of (3.9), it is possible to rearrange the series in terms of our $f$.'s. More precisely, it is simpler to use the extension $\varphi$ of $f$ described in appendix A, to zero or negative indices, generated by the series

$$
\begin{aligned}
F_{n}\left(\lambda_{1}^{-1}, . ., \lambda_{n}^{-1} ; X\right) & =\sum_{m_{i} \in \mathbb{Z}} \prod_{k=1}^{n} \lambda_{k}^{-m_{k}} \varphi_{m_{1}, . . m_{n}}(\theta .(X)) \\
& =\prod_{1 \leq i<j \leq n} \frac{\lambda_{i}^{-1}-\lambda_{j}^{-1}}{\lambda_{i}^{-1}+\lambda_{j}^{-1}} \sum_{n_{i} \geq 0} \prod_{i=1}^{n} \lambda_{i}^{-n_{i}} p_{n_{i}}(\theta .(X))
\end{aligned}
$$

expanded in the domain $\lambda_{1}^{-1}>\ldots .>\lambda_{n}^{-1}$. The l.h.s. of (3.9) reads therefore

$$
\begin{aligned}
(-1)^{\frac{n(n-1)}{2}} \prod_{1 \leq i<j \leq n} \frac{\lambda_{i}^{-1}-\lambda_{j}^{-1}}{\lambda_{i}^{-1}+\lambda_{j}^{-1}} & \Xi\left(\lambda_{1}^{-1} \oplus . . \oplus \lambda_{n}^{-1} \oplus \Lambda^{-1}\right) \\
& =(-1)^{\frac{n(n-1)}{2}} \sum_{m_{k} \in \mathbb{Z}} \prod_{k=1}^{n} \lambda_{k}^{-m_{k}} \varphi_{m_{1}, \ldots, m_{n}}(\partial .) \Xi\left(\Lambda^{-1}\right)
\end{aligned}
$$

To perform such an expansion on the r.h.s. of (3.9) we should similarly order the arguments $\lambda_{k}+\left(y_{k} / 2 i\right)$ (in modulus). This looks at first unreasonable since the $y$ 's run along the whole real axis. However we recall that we look for an asymptotic expansion of an absolutely convergent integral over $y_{1}, . ., y_{n}$, as each $\lambda_{1}, . ., \lambda_{n}$ goes to infinity. With an exponentially small error we can therefore bound the domain of integration in the $y$ 's and assume the $\lambda$ 's large enough so that the $\left|\lambda_{k}+\left(y_{k} / 2 i\right)\right|$ remain ordered. This means that in the sense of asymptotic series we can perform an expansion similar to the above (3.23) but this time with $\varphi$. $(Y / 2 i)$ in the integrand. Integrating on the $y$ 's as we did before, we can identify the coefficient of $\lambda_{1}^{-m_{1}} \ldots \lambda_{n}^{-m_{n}}, m_{1}>\ldots>m_{n}>0$ in the resulting asymptotic expansion. We obtain the main result of this section in the form of 
Proposition (W') Any polynomial in derivatives acting on $\Xi(\theta$.) can be expressed as an average over a polynomial in odd traces of $Y$ and vice versa. More precisely for $m_{1}>$.. $>$ $m_{n}>0$

$$
f_{m_{1}, . ., m_{n}}\left(\partial_{.}\right) \Xi(\theta .)=\sum_{\substack{s_{1}, \ldots, s_{n} \geq 0 \\ 3 s_{j} \leq \sum_{j \leq k \leq n} m_{k}}} \prod_{i=1}^{n}(-1)^{s_{i}} c_{s_{i}, m_{i}} \ll \varphi_{m_{1}-3 s_{1}, \ldots, m_{n}-3 s_{n}}(\theta .(Y / 2 i)) \gg_{N}
$$

We recall the notations and make a few comments.

(a) $\partial .=\left\{-\frac{2}{2 k+1} \frac{\partial}{\partial \theta_{2 k+1}}\right\}$.

(b) the $\varphi$.'s are reduced to $f$.'s with positive indices according to the rules (i)-(iv) of appendix A.

(c) $c_{s, m}=\sum_{l=0}^{2 s} \frac{1}{2^{l}}\left(\begin{array}{c}m-3 s+l-1 \\ l\end{array}\right) \frac{(6 s-2 l-1) ! !}{6^{2 s-l}(2 s-l) !}$, where the combinatorial factor can be seen as a polynomial in the variable $m-3 s$ with integral coefficients, hence remains integral and well defined for $m-3 s<0$. It is easy to rewrite

$$
c_{s, m}=\frac{1}{(12)^{2 s}} \sum_{l=0}^{2 s} 6 \frac{(6 s-2 l-1) ! !}{(4 s-2 l-1) ! !}\left(\begin{array}{c}
4 s-2 l \\
2 s-l
\end{array}\right)\left(\begin{array}{c}
m-3 s+l-1 \\
l
\end{array}\right)
$$

exhibiting $(12)^{2 s} c_{s, m}$ as an integer. Since the $\varphi$.'s are linear combinations of $f$.'s with integral coefficients (see appendix A), the relation (3.24) involves at most rational fractions with denominators $(12)^{2 s_{i}}$ as coefficients.

(d) The "leading" term in (3.24) corresponds to $s_{1}=\ldots=s_{n}=0$ and $c_{o, m}=1$, hence for $m_{1}>\ldots>m_{n}>0$

$$
f_{m_{1}, . ., m_{n}}\left(\partial_{.}\right) \Xi(\theta .)=\ll f_{m_{1}, . ., m_{n}}(\theta .(Y / 2 i)) \gg_{N}+\text { lower... }
$$

where by lower we mean averages over polynomials with smaller degree in $Y$. Therefore the system of equations (3.24) is triangular and can be inverted, vindicating the statement ( $\left.W^{\prime}\right)$.

(e) In table III we have recorded explicitely the first few cases of (3.24) up to degree 8 (the argument in the average is $\theta .(Y / 2 i)$ ). The reader can check -as we did in table IV - that these data are in agreement with formulas (3.5) of this section, expressing 
derivatives w.r.t. $\theta_{1}$ and $\theta_{3}$. Also one can make contact with earlier results by E. Witten [1] expressed in terms of variables $t$. related to our $\theta$.'s (1.4) through

$$
t_{k}=\frac{(2 k+1) ! !}{2} \theta_{2 k+1}
$$

(f) There exist simple cases of (3.24) when successive $m$ 's differ by 3 and the last one is 1 or 2 , where, due to the antisymmetry of $f$.'s, the relation (3.24) reduces to

$$
f_{a+3 k, a+3(k-1), . ., a+3, a}\left(\partial_{.}\right) \Xi(\theta .)=\ll f_{a+3 k, a+3(k-1), \ldots, a+3, a}(\theta .(Y / 2 i)) \gg_{N}
$$

for $a=1,2$. 


\section{Appendix A. Calculation of $\mathrm{f}$.'s}

To make the computation of the polynomials $f$. simple and explicit, let us use the definition of the characters ch. in terms of our Schur polynomials $p$. (2.5) to rewrite (2.27) as

$$
\begin{aligned}
f_{k_{1}, \ldots ., k_{n}}(\theta .) & =\left\{\prod_{1 \leq i<j \leq n} \frac{1-P_{k_{i}} P_{k_{j}}^{-1}}{1+P_{k_{i}} P_{k_{j}}^{-1}}\right\} p_{k_{1}}\left(\theta_{.}\right) p_{k_{2}}\left(\theta_{.}\right) \ldots p_{k_{n}}\left(\theta_{.}\right) \\
& =\sum_{\mathbf{r} \geq 0}\left(\prod_{1 \leq i<j \leq n} \alpha_{r_{i j}}\right) p_{k_{1}+\Sigma_{j} r_{1 j}}\left(\theta_{.}\right) \ldots p_{k_{n}+\Sigma_{j} r_{n j}}\left(\theta_{.}\right)
\end{aligned}
$$

where $\alpha_{r}=(-1)^{r}\left(2-\delta_{r, 0}\right)$ is the coefficient of $y^{r}$ in the small $y$ expansion of $(1-y) /(1+y)$, and the sum over $\mathbf{r}$ (defined as in (2.27)) is finite $\left(k_{p}+\sum_{j} r_{p j} \geq 0\right)$. The second line of (A.1) makes it straightforward to extend the definition of $f$.'s to $\varphi$.'s including negative or zero indices. Namely define for $m_{1}, . ., m_{n} \in \mathbb{Z}$

$$
\varphi_{m_{1}, . ., m_{n}}=\sum_{\mathbf{r} \geq 0}\left(\prod_{1 \leq i<j \leq n} \alpha_{r_{i j}}\right) p_{m_{1}+\sum_{i} r_{1 i} \ldots p_{m_{n}}+\sum_{i} r_{n i}}
$$

with the convention that $p_{m}=0$ as soon as $m<0$, then one has obviously

$$
\varphi_{m_{1}, . ., m_{n}}=f_{m_{1}, \ldots, m_{n}} \quad \text { for } m_{1}, \ldots, m_{n}>0
$$

This definition enables one to rewrite the expressions (2.23) (2.24) for the characters in a very simple way

$$
\text { ch. }=\prod_{i<j}\left(P_{i}^{-1}+P_{j}^{-1}\right) \varphi .
$$

The generating function (2.19) for characters also simplifies drastically. Namely in the region $\left|u_{1}\right|>\left|u_{2}\right|>\ldots>\left|u_{n}\right|$ of the polydisk $D(X)^{n}$, we can expand $F$ defined in (2.8) as

$$
\begin{aligned}
F_{n}\left(u_{1}, \ldots, u_{n} ; X\right) & =\prod_{1 \leq i<j \leq n} \frac{1-\left(u_{j} / u_{i}\right)}{1+\left(u_{j} / u_{i}\right)} \prod_{k=1}^{n} \operatorname{det} \frac{1-u_{k} X}{1+u_{k} X} \\
& =\sum_{\mathbf{r} \geq 0} \sum_{k_{1}, \ldots, k_{n} \geq 0} \prod_{1 \leq i<j \leq n} \alpha_{r_{i j}}\left(u_{j} / u_{i}\right)^{r_{i j}} \prod_{m=1}^{n} u_{m}^{k_{m}} p_{k_{m}+\sum_{j} r_{m j}}(X) \\
& =\sum_{m_{1}, . ., m_{n} \in \mathbb{Z}}\left(\prod_{i=1}^{n} u_{i}^{m_{i}}\right) \varphi_{m_{1}, \ldots, m_{n}}(X)
\end{aligned}
$$


But as shown in section 2, the $f$.'s form a basis of the vector space of polynomials in the variable $\theta$., therefore the extension $\varphi$. is overcomplete. In fact as already apparent on the r.h.s. of (2.19), the $\varphi$.'s can be expressed simply in terms of $f$.'s only: we get the following set of rules easily derived from the definition (A.2)

(i) $\varphi_{m_{1}, \ldots, m_{n}}=f_{m_{1}, \ldots, m_{n}}$, for all $m_{1}>0$.

(ii) $\varphi_{m_{1}, \ldots, m_{n}, 0}=\varphi_{m_{1}, \ldots, m_{n}}$, for all $m_{i} \in \mathbb{Z}$.

(iii) $\varphi_{m_{1}, \ldots, m_{n}, a}=0$, whenever $a<0$.

(iv) $\varphi_{m_{1}, . ., m_{j+1}, m_{j}, . ., m_{n}}=-\varphi_{m_{1}, . ., m_{j}, m_{j+1}, . ., m_{n}}+2(-1)^{m_{j}} \delta_{m_{j}+m_{j+1}, 0} \varphi_{m_{1}, . ., \widehat{m_{j}}, \widehat{m_{j+1}}, . ., m_{n}}$. The only non-trivial rule is the last one, which follows from the identity

$$
\sum_{0 \leq r \leq m}(-1)^{r} \alpha_{r} \alpha_{m-r}=\delta_{m, 0}
$$

Starting from some $\varphi_{m_{1}, . ., m_{n}}$, to reexpress it in terms of $f$.' $\mathrm{s}$ one has to use the rule (iv) repeatedly to "push" the negative indices to the right, which results in either (ii) or (iii), and ends up with an expression of $f$ through (i). Note that in this way $\varphi$.'s are expressed as linear combinations of the $f$.'s with relative integer coefficients. We have for instance

$$
\begin{aligned}
& \varphi_{m_{1},-m_{2}, m_{3}}=2(-1)^{m_{2}} \delta_{m_{2}, m_{3}} f_{m_{1}} \quad m_{1}, m_{2}, m_{3}>0 \\
& \varphi_{-m_{1}, m_{2}, m_{3}}=2(-1)^{m_{1}}\left[\delta_{m_{1}, m_{2}} f_{m_{3}}-\delta_{m_{1}, m_{3}} f_{m_{2}}\right] m_{1}, m_{2}, m_{3}>0
\end{aligned}
$$

which should be compared with (2.24).

We now turn to the actual computation of the $f$.'s in terms of $\theta$.'s. From the last equality in (A.1), we get a recursion relation for $f$.'s

$$
f_{k_{1}, \ldots, k_{n+1}}(\theta .)=\sum_{s=0}^{k_{n+1}} p_{k_{n+1}-s} \sum_{\substack{r_{1}, \ldots, r_{n} \geq 0 \\ r_{1}+\ldots+r_{n}=s}}\left(\prod_{i=1}^{n} \alpha_{r_{i}}\right) f_{k_{1}+r_{1}, \ldots, k_{n}+r_{n}}(\theta .)
$$

Let us use the shorthand notation

$$
\theta_{\{\nu\}}=\theta_{\left[1^{\nu_{1}} 3^{\left.\nu_{3} \ldots\right]}\right.}=\prod_{\text {odd }} \frac{\theta_{j}^{\nu_{j}}}{\nu_{j} !}
$$

for any set $\{\nu\}=\nu_{1}, \nu_{3}, \ldots$ of non negative integers (or alternatively any permutation $\left[1^{\nu_{1}} 3^{\nu_{3}} \ldots\right]$ of $\sum_{\text {odd } j} j \nu_{j}$ elements with odd cycles only), then the coefficients $A$ in the expansion

$$
f_{k_{1}, \ldots, k_{n}}(\theta .)=\sum_{\substack{\nu_{j} \geq 0, j \text { odd } \\ \nu_{1}+3 \nu_{3}+\ldots=k_{1}+\ldots+k_{n}}} A_{k_{1}, \ldots, k_{n}}^{\{\nu\}} \theta_{\{\nu\}}
$$


satisfy the recursion relation

$$
\begin{aligned}
& A_{k_{1}, \ldots, k_{n+1}}^{\{\nu\}}= \sum_{\substack{\mu_{j} \geq 0, j \text { odd } \\
\mu_{j} \leq \nu_{j} ;\left(\nu_{1}-\mu_{1}\right)+3\left(\nu_{3}-\mu_{3}\right)+\ldots \leq k_{n+1}}} \prod_{j \text { odd }}\left(\begin{array}{l}
\nu_{j} \\
\mu_{j}
\end{array}\right) \times \\
& \times \sum_{\substack{r_{1}, \ldots, r_{n} \geq 0 \\
r_{1}+\ldots+r_{n}=k_{n+1}+\left(\mu_{1}-\nu_{1}\right)+3\left(\mu_{3}-\nu_{3}\right)+\ldots}}^{n}\left(\prod_{i=1}^{n} \alpha_{r_{i}}\right) A_{k_{1}+r_{1}, \ldots, k_{n}+r_{n}}^{\{\mu\}}
\end{aligned}
$$

Considering that only integer coefficients enter the recursion relation and that $A_{k}^{\{\nu\}}=$ 1 for $\sum_{j \text { odd }} j \nu_{j}=k$, we deduce that all $A$ 's are integers. It is now easy to compute the first few $A$ 's, we find (the multi-index superscript $\{\nu\}$ is always related to the indices through $\left.\sum_{j} j \nu_{j}=\sum k_{i}\right)$

$$
\begin{aligned}
A_{k}^{\{\nu\}} & =1 \\
A_{k, 1}^{\{\nu\}} & =\nu_{1}-2 \\
A_{k, 2}^{\{\nu\}} & =\frac{\left(\nu_{1}-1\right)\left(\nu_{1}-4\right)}{2 !} \\
A_{k, 3}^{\{\nu\}} & =\frac{\left(\nu_{1}-1\right)\left(\nu_{1}-2\right)\left(\nu_{1}-6\right)}{3 !}+\nu_{3} \\
A_{k, 4}^{\{\nu\}} & =\frac{\left(\nu_{1}-1\right)\left(\nu_{1}-2\right)\left(\nu_{1}-3\right)\left(\nu_{1}-8\right)}{4 !}+\left(\nu_{1}-2\right) \nu_{3} \\
A_{k, 5}^{\{\nu\}} & =\frac{\left(\nu_{1}-1\right)\left(\nu_{1}-2\right)\left(\nu_{1}-3\right)\left(\nu_{1}-4\right)\left(\nu_{1}-10\right)}{5 !}+\frac{\left(\nu_{1}-1\right)\left(\nu_{1}-4\right)}{2 !} \nu_{3}+\nu_{5} \\
A_{k, 2,1}^{\{\nu\}} & =\frac{\nu_{1}\left(\nu_{1}-4\right)\left(\nu_{1}-5\right)}{3 !}-2 \nu_{3} \\
A_{k, 3,1}^{\{\nu\}} & =2 \frac{\nu_{1}\left(\nu_{1}-2\right)\left(\nu_{1}-5\right)\left(\nu_{1}-7\right)}{4 !}-\left(\nu_{1}-2\right) \nu_{3} \\
A_{k, 4,1}^{\{\nu\}} & =3 \frac{\nu_{1}\left(\nu_{1}-2\right)\left(\nu_{1}-3\right)\left(\nu_{1}-6\right)\left(\nu_{1}-9\right)}{5 !}-2 \nu_{5} \\
A_{k, 3,2}^{\{\nu\}} & =2 \frac{\nu_{1}\left(\nu_{1}-1\right)\left(\nu_{1}-4\right)\left(\nu_{1}-7\right)\left(\nu_{1}-8\right)}{5 !}-\frac{\left(\nu_{1}-1\right)\left(\nu_{1}-4\right)}{2} \nu_{3}+2 \nu_{5}
\end{aligned}
$$

Table I is obtained by using these expressions. 


\section{Appendix B. Two illustrative cases}

For any invariant polynomial $f(Y)$, i.e. depending only on the eigenvalues of $Y$, one may write following the steps of [3]

$$
\begin{aligned}
& \ll f(Y+i \Lambda) \gg=\prod_{i} \lambda_{i}^{\frac{1}{2}} \prod_{i<j}\left(\lambda_{i}+\lambda_{j}\right) \int d Y f(Y+i \Lambda) e^{\frac{i}{6} \operatorname{tr} Y^{3}-\frac{1}{2} \operatorname{tr} Y^{2} \Lambda} \\
& =\prod_{i} \lambda_{i}^{\frac{1}{2}} \prod_{i<j}\left(\lambda_{i}+\lambda_{j}\right) e^{\frac{1}{6} \operatorname{tr} \Lambda^{3}} \int d Y f(Y) e^{i\left(\frac{1}{6} \operatorname{tr} Y^{3}+\frac{1}{2} \operatorname{tr} Y \Lambda^{2}\right)} \\
& =\frac{1}{N !} \int \prod_{i} \frac{d y_{i}}{\sqrt{2 \pi}} f\left(y_{j}\right) e^{\frac{1}{3} \sum \lambda_{i}^{3}+i \sum \frac{1}{6} y_{i}^{3}+\frac{1}{2} y_{i} \lambda_{i}^{2}} \prod \lambda_{i}^{\frac{1}{2}} \prod_{i<j}\left(\frac{y_{i}-y_{j}}{\lambda_{j}-\lambda_{i}}\right) \\
& =\frac{1}{N !} \int \prod_{i} \frac{d y_{i}}{\sqrt{2 \pi}}\left(f\left(-2 i \frac{\partial}{\partial \lambda_{j}^{2}}\right) e^{i \sum \frac{1}{6} y_{i}^{3}+\frac{1}{2} y_{i} \lambda_{i}^{2}}\right) e^{\frac{1}{3} \sum \lambda_{i}^{3}} \prod \lambda_{i}^{\frac{1}{2}} \frac{\prod_{i<j}\left(y_{i}-y_{j}\right)}{\Delta(\lambda)} \\
& =\frac{1}{N ! \Delta(\lambda)} f\left(i \lambda_{j}^{\frac{1}{2}} e^{\frac{1}{3} \lambda_{j}^{3}}\left(-2 \frac{\partial}{\partial \lambda_{j}^{2}}\right) \lambda_{j}^{-\frac{1}{2}} e^{-\frac{1}{3} \lambda_{j}^{3}}\right) \int \prod \frac{d y_{i}}{\sqrt{2 \pi}} \lambda_{i}^{\frac{1}{2}} e^{\frac{1}{3} \lambda_{i}^{3}} \prod_{i<j}\left(y_{i}-y_{j}\right) e^{i \sum \frac{1}{6} y_{i}^{3}+\frac{1}{2} y_{i} \lambda_{i}^{2}} \\
& =\frac{1}{\Delta(\lambda)} f(i D) \Delta(\lambda) \Xi \text {. }
\end{aligned}
$$

where the double bracket denotes the weighted average (1.5), $\Delta(\lambda)$ the Vandermonde determinant of the $\lambda$ 's and

$$
\begin{aligned}
D_{i} & =\lambda_{i}+\frac{2}{\lambda_{i}}-\frac{1}{\lambda_{i}} \frac{\partial}{\partial \lambda_{i}} \\
& =-e^{\frac{1}{3} \lambda_{i}^{3}} \lambda_{i}^{\frac{1}{2}} \frac{2 \partial}{\partial \lambda_{i}^{2}} \lambda_{i}^{-\frac{1}{2}} e^{-\frac{1}{3} \lambda_{i}^{3}} .
\end{aligned}
$$

The second equality in (B.1) is obtained by a translation $Y \rightarrow Y-i \Lambda$ for an analytic continuation to pure imaginary $\Lambda$ (see section 3 ). The next one follows from an angular integration.

The above relation is in particular true for arbitrary powers of $\operatorname{tr}(Y+i \Lambda)$

$$
\ll\left[\operatorname{tr}\left(\frac{Y}{i}+\Lambda\right)\right]^{p} \gg=\frac{1}{\Delta(\lambda)}[\operatorname{tr} D]^{p} \Delta(\lambda) \Xi .
$$

One forms a generating function for the averages of powers of $\operatorname{tr} Y$ in the form

$$
\begin{aligned}
\ll e^{s \operatorname{tr} \frac{Y}{i}} \gg & =e^{-s \operatorname{tr} \Lambda} \ll e^{s \operatorname{tr}\left(\frac{Y}{i}+\Lambda\right)} \gg \\
& =e^{-s \operatorname{tr} \Lambda} \frac{1}{\Delta(\lambda)} e^{s \operatorname{tr} D} \Delta(\lambda) \Xi \\
& =e^{-s \operatorname{tr} \Lambda}\left(\frac{1}{\Delta(\lambda)} e^{\frac{1}{3} \operatorname{tr} \Lambda^{3}} \operatorname{det} \Lambda^{\frac{1}{2}}\right) e^{-2 s \sum \frac{\partial}{\partial \lambda_{i}^{2}}}\left(\operatorname{det} \Lambda^{-\frac{1}{2}} e^{-\frac{1}{3} \operatorname{tr} \Lambda^{3}} \Delta(\lambda) \Xi\right) .
\end{aligned}
$$


The operator $e^{-2 s \sum \frac{\partial}{\partial \lambda_{i}^{2}}}$ shifts all variables $\lambda_{i}^{2}$ by $-2 s$. Hence, using the expressions displayed in [3], one finds after some algebra

$$
\ll e^{s \operatorname{tr} \frac{Y}{i}} \gg=e^{\psi(s)} \exp s \sum_{n \geq 0} t_{n+1} \frac{\partial}{\partial t_{n}} \Xi
$$

where the variables $t$. are related to our $\theta$. defined in (1.4) through

$$
t_{k}=\frac{(2 k+1) ! !}{2} \theta_{2 k+1}
$$

and $\psi$ is the function given by

$$
\psi(s)=\frac{1}{3} \sum \lambda_{i}^{3}-\frac{1}{3} \sum\left(\lambda_{i}^{2}-2 s\right)^{\frac{1}{2}}+\sum_{i<j} \ln \frac{\lambda_{i}+\lambda_{j}}{\sqrt{\lambda_{i}^{2}-2 s}+\sqrt{\lambda_{j}^{2}-2 s}}-\frac{1}{4} \ln \frac{\lambda_{i}^{2}-2 s}{\lambda_{i}^{2}}-s \sum \lambda_{i} .
$$

The differential operator $l_{-1}$ that appears in the exponential of the r.h.s. of (B.5) is part of a Virasoro operator that annihilates the function $\Xi$, namely $L_{-1}=\sum_{n \geq 0} t_{n+1} \frac{\partial}{\partial t_{n}}+\frac{1}{2} t_{0}^{2}-\frac{\partial}{\partial t_{0}}$. This may be used to rewrite $(\overline{B .5})$ as

$$
\ll e^{s \operatorname{tr} \frac{Y}{i}} \gg=e^{\psi(s)} e^{s l_{-1}} e^{-s L_{-1}} \Xi
$$

Denoting $K(s)=e^{\psi(s)} e^{s l_{-1}} e^{-s L_{-1}}$, one finds that $\frac{\partial}{\partial s} K(s)=\left(\frac{\partial}{\partial t_{0}}-\frac{1}{2} s^{2}\right) K(s)$ whence

$$
\ll e^{s \operatorname{tr} \frac{Y}{i}} \gg=e^{s \frac{\partial}{\partial t_{0}}-\frac{s^{3}}{6}} \Xi
$$

or equivalently

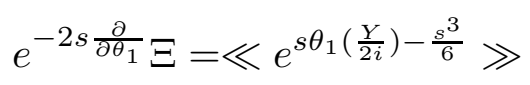

in terms of our $\theta$.'s. Therefore in this particular case, we have a very explicit expression of correlation functions in terms of derivatives of $\Xi$, vindicating the general proposition (W).

The averages of powers of $\operatorname{tr} Y^{3}$ may also be treated simply. By a rescaling of the integration variable $Y$, it is easy to derive

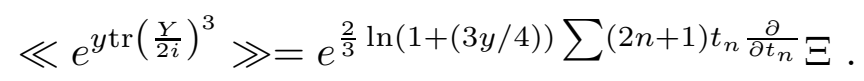

Here too, the differential operator $l_{0}=\sum(2 n+1) t_{n} \frac{\partial}{\partial t_{n}}$ in the exponential is a part of the Virasoro generator $L_{0}=l_{0}+\frac{1}{16}-\frac{3}{2} \frac{\partial}{\partial t_{1}}$ that annihilates $\Xi$. As above, we define $K^{\prime}(z)=e^{2 z l_{0}} e^{-2 z L_{0}}$ and compute that

$$
\frac{\partial}{\partial z} K^{\prime}(z)=\left(3 e^{-3 z} \frac{\partial}{\partial t_{1}}-\frac{1}{8}\right) K^{\prime}(z)
$$


Thus $K^{\prime}(z)=\exp -\left[\left(e^{-3 z}-1\right) \frac{\partial}{\partial t_{1}}+\frac{z}{8}\right]$. This leads to

$$
\ll e^{y \operatorname{tr}\left(\frac{Y}{2 i}\right)^{3}} \gg=(1+(3 y / 4))^{-\frac{1}{12}} e^{-\left[(1+(3 y / 4))^{-2}-1\right] \frac{\partial}{\partial t_{1}}} \Xi
$$

showing again that any $\ll\left[\operatorname{tr}\left(\frac{Y}{2 i}\right)^{3}\right]^{p} \gg$ is given by a polynomial in the derivative $\frac{\partial}{\partial t_{1}}$ acting on $\Xi$. The two special cases $(\mathbb{B} .9)$ and $(\mathbb{B} .12)$ may be combined into

$$
\ll e^{s \operatorname{tr} \frac{Y}{i}+y \operatorname{tr}\left(\frac{Y}{2 i}\right)^{3}} \gg=\exp \left(\tilde{s} \frac{\partial}{\partial \tilde{t}_{0}}-\frac{\tilde{s}^{3}}{6}\right) \Xi\left(\tilde{\Lambda}^{-1}\right)
$$

where $\tilde{\Lambda}=(y / 8)^{-\frac{2}{3}} \Lambda$, hence $\tilde{t}_{n}=(y / 8)^{\frac{2}{3}(2 n+1)} t_{n}$ and $\tilde{s}=(y / 8)^{-\frac{1}{3}} s$. 


\section{Table I}

\begin{tabular}{|c|c|}
\hline$f_{1}$ & $=\theta_{\left[1^{1}\right]}$ \\
\hline$f_{2}$ & $=\theta_{\left[1^{2}\right]}$ \\
\hline$f_{3}$ & $=\theta_{\left[1^{3}\right]}+\theta_{\left[3^{1}\right]}$ \\
\hline$f_{2,1}$ & $=\theta_{\left[1^{3}\right]}-2 \theta_{\left[3^{1}\right]}$ \\
\hline$f_{4}$ & $=\theta_{\left[1^{4}\right]}+\theta_{\left[1^{1} 3^{1}\right]}$ \\
\hline$f_{3,1}$ & $=2 \theta_{\left[1^{4}\right]}-\theta_{\left[1^{1} 3^{1}\right]}$ \\
\hline$f_{5}$ & $=\theta_{\left[1^{5}\right]}+\theta_{\left[1^{2} 3^{1}\right]}+\theta_{\left[5^{1}\right]}$ \\
\hline$f_{4,1}$ & $=3 \theta_{\left[1^{5}\right]}-2 \theta_{\left[5^{1}\right]}$ \\
\hline$f_{3,2}$ & $=2 \theta_{\left[1^{5}\right]}-\theta_{\left[1^{2} 3^{1}\right]}+2 \theta_{\left[5^{1}\right]}$ \\
\hline$f_{6}$ & $=\theta_{\left[1^{6}\right]}+\theta_{\left[1^{3} 3^{1}\right]}+\theta_{\left[1^{1} 5^{1}\right]}+\theta_{\left[3^{2}\right]}$ \\
\hline$f_{5,1}$ & $=4 \theta_{\left[1^{6}\right]}+\theta_{\left[1^{3} 3^{1}\right]}-\theta_{\left[1^{1} 5^{1}\right]}-2 \theta_{\left[3^{2}\right]}$ \\
\hline$f_{4,2}$ & $=5 \theta_{\left[1^{6}\right]}-\theta_{\left[1^{3} 3^{1}\right]}+2 \theta_{\left[3^{2}\right]}$ \\
\hline$f_{3,2,1}$ & $=2 \theta_{\left[1^{6}\right]}-\theta_{\left[1^{3} 3^{1}\right]}+2 \theta_{\left[1^{1} 5^{1}\right]}-4 \theta_{\left[3^{2}\right]}$ \\
\hline$f_{7}$ & $=\theta_{\left[1^{7}\right]}+\theta_{\left[1^{4} 3^{1}\right]}+\theta_{\left[1^{2} 5^{1}\right]}+\theta_{\left[1^{1} 3^{2}\right]}+\theta_{\left[7^{1}\right]}$ \\
\hline$f_{6,1}$ & $=5 \theta_{\left[1^{7}\right]}+2 \theta_{\left[1^{4} 3^{1}\right]}-\theta_{\left[1^{1} 3^{2}\right]}-2 \theta_{\left[7^{1}\right]}$ \\
\hline$f_{5,2}$ & $=9 \theta_{\left[1^{7}\right]}-\theta_{\left[1^{2} 5^{1}\right]}+2 \theta_{\left[7^{1}\right]}$ \\
\hline$f_{4,3}$ & $=5 \theta_{\left[1^{7}\right]}-\theta_{\left[1^{4} 3^{1}\right]}+2 \theta_{\left[1^{1} 3^{2}\right]}-2 \theta_{\left[7^{1}\right]}$ \\
\hline$f_{4,2,1}$ & $=7 \theta_{\left[1^{7}\right]}-2 \theta_{\left[1^{4} 3^{1}\right]}+2 \theta_{\left[1^{2} 5^{1}\right]}-2 \theta_{\left[1^{1} 3^{2}\right]}$ \\
\hline$f_{8}$ & $=\theta_{\left[1^{8}\right]}+\theta_{\left[1^{5} 3^{1}\right]}+\theta_{\left[1^{3} 5^{1}\right]}+\theta_{\left[1^{2} 3^{2}\right]}+\theta_{\left[1^{1} 7^{1}\right]}+\theta_{\left[3^{1} 5^{1}\right]}$ \\
\hline$f_{7,1}$ & $=6 \theta_{\left[1^{8}\right]}+3 \theta_{\left[1^{5} 3^{1}\right]}+\theta_{\left[1^{3} 5^{1}\right]}-\theta_{\left[1^{1} 7^{1}\right]}-2 \theta_{\left[3^{1} 5^{1}\right]}$ \\
\hline$f_{6,2}$ & $=14 \theta_{\left[1^{8}\right]}+2 \theta_{\left[1^{5} 3^{1}\right]}-\theta_{\left[1^{3} 5^{1}\right]}-\theta_{\left[1^{2} 3^{2}\right]}+2 \theta_{\left[3^{1} 5^{1}\right]}$ \\
\hline$f_{5,3}$ & $=14 \theta_{\left[1^{8}\right]}-\theta_{\left[1^{5} 3^{1}\right]}-\theta_{\left[1^{3} 5^{1}\right]}+2 \theta_{\left[1^{2} 3^{2}\right]}-\theta_{\left[3^{1} 5^{1}\right]}$ \\
\hline$f_{5,2,1}$ & $=16 \theta_{\left[1^{8}\right]}-2 \theta_{\left[1^{5} 3^{1}\right]}+\theta_{\left[1^{3} 5^{1}\right]}-2 \theta_{\left[1^{2} 3^{2}\right]}+2 \theta_{\left[1^{1} 7^{1}\right]}-2 \theta_{\left[3^{1} 5^{1}\right]}$ \\
\hline$f_{4,3,1}$ & $=12 \theta_{\left[1^{8}\right]}-3 \theta_{\left[1^{5} 3^{1}\right]}+2 \theta_{\left[1^{3} 5^{1}\right]}-2 \theta_{\left[1^{1} 7^{1}\right]}+2 \theta_{\left[3^{1} 5^{1}\right]}$ \\
\hline
\end{tabular}

The $f$ polynomials up to degree 8 . The notation $\theta_{\left[1^{\nu_{1}} 3^{\nu_{3}} \ldots(2 k+1)^{\nu_{2 k+1}} . .\right]}$ is a short hand for $\frac{\theta_{1}^{\nu_{1}}}{\nu_{1} !} \frac{\theta_{3}^{\nu_{3}}}{\nu_{3} !} \cdot . \frac{\theta_{2 k+1}^{\nu_{2 k+1}}}{\nu_{2 k+1} !} \ldots$ 
Table II

\begin{tabular}{|l}
$\theta_{\left[1^{1}\right]}=f_{1}$ \\
\hline$\theta_{\left[1^{2}\right]}=f_{2}$ \\
\hline$\theta_{\left[1^{3}\right]}=\frac{1}{3}\left[2 f_{3}+f_{2,1}\right]$ \\
$\theta_{\left[3^{1}\right]}=\frac{1}{3}\left[f_{3}-f_{2,1}\right]$ \\
\hline$\theta_{\left[1^{4}\right]}=\frac{1}{3}\left[f_{4}+f_{3,1}\right]$ \\
$\theta_{\left[1^{1} 3^{1}\right]}=\frac{1}{3}\left[2 f_{4}-f_{3,1}\right]$ \\
\hline$\theta_{\left[1^{5}\right]}=\frac{1}{15}\left[2 f_{5}+3 f_{4,1}+2 f_{3,2}\right]$ \\
$\theta_{\left[1^{2} 3^{1}\right]}=\frac{1}{3}\left[2 f_{5}-f_{3,2}\right]$ \\
$\theta_{\left[5^{1}\right]}=\frac{1}{5}\left[f_{5}-f_{4,1}+f_{3,2}\right]$ \\
\hline$\theta_{\left[1^{6}\right]}=\frac{1}{45}\left[2 f_{6}+4 f_{5,1}+5 f_{4,2}+f_{3,2,1}\right]$ \\
$\theta_{\left[1^{3} 3^{1}\right]}=\frac{1}{9}\left[4 f_{6}+2 f_{5,1}-2 f_{4,2}-f_{3,2,1}\right]$ \\
$\theta_{\left[1^{1} 5^{1}\right]}=\frac{1}{5}\left[2 f_{6}-f_{5,1}+f_{3,2,1}\right]$ \\
$\theta_{\left[3^{2}\right]}=\frac{1}{9}\left[f_{6}-f_{5,1}+f_{4,2}-f_{3,2,1}\right]$ \\
\hline$\theta_{\left[1^{7}\right]}=\frac{1}{315}\left[4 f_{7}+10 f_{6,1}+18 f_{5,2}+10 f_{4,3}+7 f_{4,2,1}\right]$ \\
$\theta_{\left[1^{4} 3^{1}\right]}=\frac{1}{9}\left[2 f_{7}+2 f_{6,1}-f_{4,3}-f_{4,2,1}\right]$ \\
$\theta_{\left[1^{2} 5^{1}\right]}=\frac{1}{5}\left[2 f_{7}-f_{5,2}+f_{4,2,1}\right]$ \\
$\theta_{\left[1^{1} 3^{2}\right]}=\frac{1}{9}\left[2 f_{7}-f_{6,1}+2 f_{4,3}-f_{4,2,1}\right]$ \\
$\theta_{\left[7^{1}\right]}=\frac{1}{7}\left[f_{7}+f_{5,2}-f_{6,1}-f_{4,3}\right]$ \\
\hline$\theta_{\left[1^{8}\right]}=\frac{1}{315}\left[f_{8}+3 f_{7,1}+7 f_{6,2}+7 f_{5,3}+4 f_{5,2,1}+3 f_{4,3,1}\right]$ \\
$\theta_{\left[1^{5} 3^{1}\right]}=\frac{1}{45}\left[4 f_{8}+6 f_{7,1}+4 f_{6,2}-2 f_{5,3}-2 f_{5,2,1}-3 f_{4,3,1}\right]$ \\
$\theta_{\left[1^{3} 5^{1}\right]}=\frac{1}{15}\left[4 f_{8}+2 f_{7,1}-2 f_{6,2}-2 f_{5,3}+f_{5,2,1}+2 f_{4,3,1}\right]$ \\
$\theta_{\left[1^{2} 3^{2}\right]}=\frac{1}{9}\left[2 f_{8}-f_{6,2}+2 f_{5,3}-f_{5,2,1}\right]$ \\
$\theta_{\left[1^{1} 7^{1}\right]}=\frac{1}{7}\left[2 f_{8}-f_{7,1}+f_{5,2,1}-f_{4,3,1}\right]$ \\
$\theta_{\left[3^{1} 5^{1}\right]}=\frac{1}{15}\left[2 f_{8}-2 f_{7,1}+2 f_{6,2}-f_{5,3}-f_{5,2,1}+f_{4,3,1}\right]$ \\
\end{tabular}

Expression of $\theta$. monomials in terms of $f$.'s up to degree 8 . 


\section{Table III}

\begin{tabular}{|c|c|}
\hline$f_{1}(\partial.) \Xi$ & $=\ll f_{1} \gg$ \\
\hline$f_{2}(\partial.) \Xi$ & $=\ll f_{2} \gg$ \\
\hline$f_{3}(\partial.) \Xi$ & $=\ll f_{3}-\frac{5}{24} \gg$ \\
\hline$f_{2,1}(\partial.) \Xi$ & $=\ll f_{2,1}-\frac{1}{12} \gg$ \\
\hline$f_{4}(\partial.) \Xi$ & $=\ll f_{4}-\frac{17}{24} f_{1} \gg$ \\
\hline$f_{3,1}(\partial.) \Xi$ & $=\ll f_{3,1}+\frac{5}{24} f_{1} \gg$ \\
\hline$f_{5}(\partial.) \Xi$ & $=\ll f_{5}-\frac{35}{24} f_{2} \gg$ \\
\hline$f_{4,1}(\partial.) \Xi$ & $=\ll f_{4,1} \gg$ \\
\hline$f_{3,2}(\partial.) \Xi$ & $=\ll f_{3,2}+\frac{5}{24} f_{2} \gg$ \\
\hline$f_{6}(\partial.) \Xi$ & $=\ll f_{6}-\frac{59}{24} f_{3}+\frac{385}{1152} \gg$ \\
\hline$f_{5,1}(\partial.) \Xi$ & $=\ll f_{5,1}-\frac{35}{24} f_{2,1}+\frac{35}{576} \gg$ \\
\hline$f_{4,2}(\partial.) \Xi$ & $=\ll f_{4,2}+\frac{17}{24} f_{2,1}-\frac{35}{576} \gg$ \\
\hline$f_{3,2,1}(\partial.) \Xi$ & $=\ll f_{3,2,1}-\frac{5}{24} f_{2,1}-\frac{1}{12} f_{3}+\frac{5}{288} \gg$ \\
\hline$f_{7}(\partial.) \Xi$ & $=\ll f_{7}-\frac{89}{24} f_{4}+\frac{1801}{1152} f_{1} \gg$ \\
\hline$f_{6,1}(\partial.) \Xi$ & $=\ll f_{6,1}-\frac{59}{24} f_{3,1}-\frac{385}{1152} f_{1} \gg$ \\
\hline$f_{5,2}(\partial.) \Xi$ & $=\ll f_{5,2} \gg$ \\
\hline$f_{4,3}(\partial.) \Xi$ & $=\ll f_{4,3}+\frac{17}{24} f_{3,1}-\frac{5}{24} f_{4}+\frac{85}{576} f_{1} \gg$ \\
\hline$f_{4,2,1}(\partial.) \Xi$ & $=\ll f_{4,2,1}-\frac{1}{12} f_{4}-\frac{1}{576} f_{1} \gg$ \\
\hline$f_{8}(\partial.) \Xi$ & $=\ll f_{8}-\frac{125}{24} f_{5}+\frac{5005}{1152} f_{2} \gg$ \\
\hline$f_{7,1}(\partial.) \Xi$ & $=\ll f_{7,1}-\frac{89}{24} f_{4,1} \gg$ \\
\hline$f_{6,2}(\partial.) \Xi$ & $=\ll f_{6,2}-\frac{59}{24} f_{3,2}-\frac{385}{1152} f_{2} \gg$ \\
\hline$f_{5,3}(\partial.) \Xi$ & $=\ll f_{5,3}+\frac{35}{24} f_{3,2}-\frac{5}{24} f_{5}+\frac{175}{576} f_{2} \gg$ \\
\hline$f_{5,2,1}(\partial.) \Xi$ & $=\ll f_{5,2,1}-\frac{1}{12} f_{5}+\frac{35}{576} f_{2} \gg$ \\
\hline$f_{4,3,1}(\partial.) \Xi$ & $=\ll f_{4,3,1}+\frac{5}{24} f_{4,1} \gg$ \\
\hline
\end{tabular}

The derivatives of the Kontsevich partition function with respect to the $\theta$.'s expressed as averages over polynomials in odd traces. The notation $\partial$. stands for $\left\{-\frac{2}{2 k+1} \frac{\partial}{\partial \theta_{2 k+1}}\right\}$, $\theta$. $\equiv \theta\left(\Lambda^{-1}\right)$, while on the r.h.s. the matrix argument of the $f$.'s is $Y / 2 i$. 
Table IV

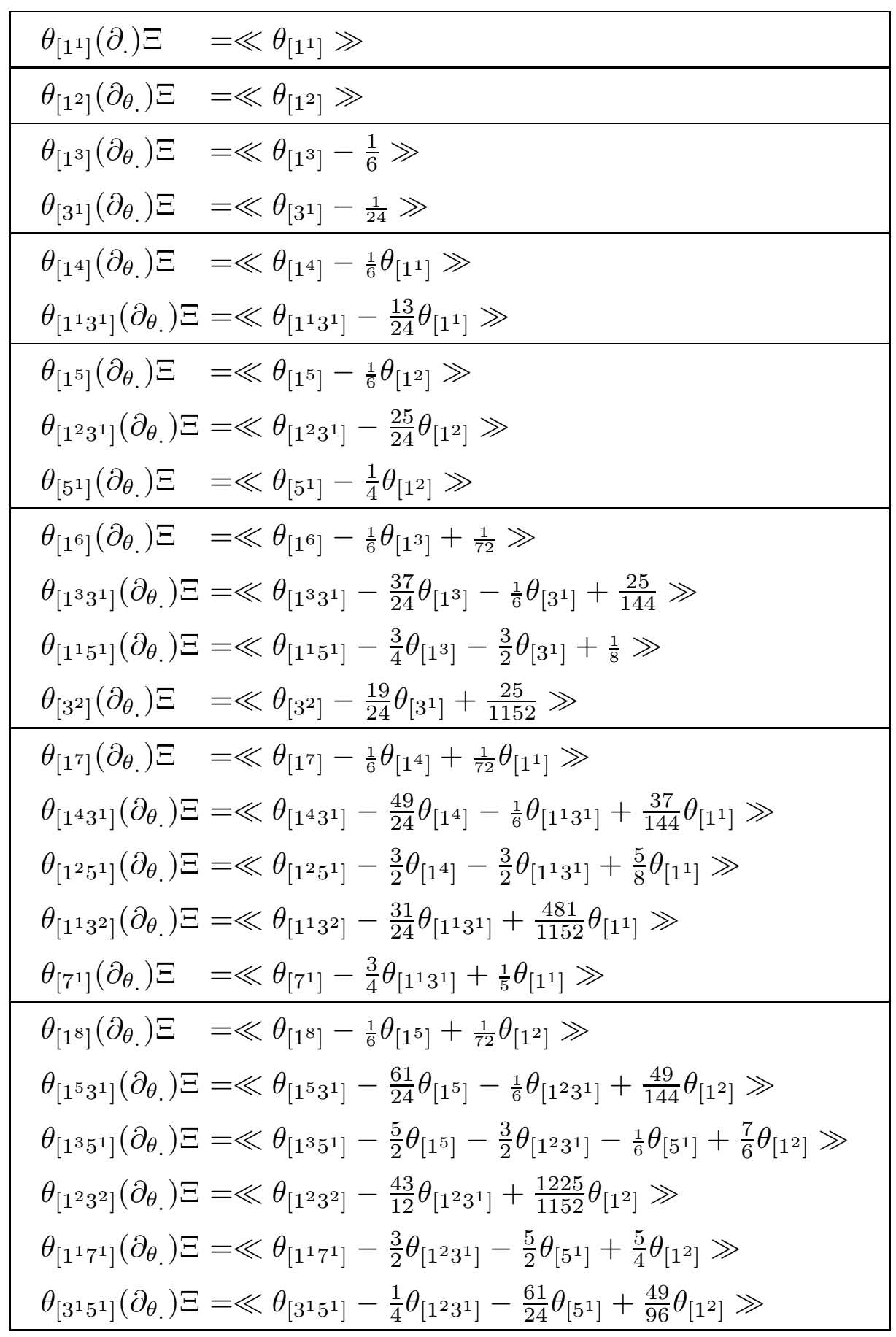

Monomials in the derivatives acting on the Kontsevich integral expressed as averages of polynomials. The notation $\theta_{\left[\ldots(2 k+1)^{\left.\nu_{2 k+1} \ldots\right]}\right.}\left(\partial_{\theta}\right)$ stands for $\ldots \frac{1}{\nu_{2 k+1} !} \partial_{2 k+1}^{\nu_{2 k+1}} \ldots \equiv$ $\cdots \frac{1}{\nu_{2 k+1} !}\left(-(2 / 2 k+1) \partial_{\theta_{2 k+1}}\right)^{\nu_{2 k+1}} \cdots$ 


\section{References}

[1] E. Witten, On the Kontsevich model and other models of two dimensional gravity, preprint IASSNS-HEP-91/24

[2] M. Kontsevich, Intersection theory on the moduli space of curves, Funk. Anal.\& Prilozh., 25 (1991) 50-57;

Intersection theory on the moduli space of curves and the matrix Airy function, lecture at the Arbeitstagung, Bonn, June 1991 and Bonn preprint MPI/91-77.

[3] C. Itzykson and J.-B. Zuber, Combinatorics of the Modular Group II: The Kontsevich integrals., to appear in Int. J. Mod. Phys.

[4] E. Witten and M. Kontsevich, private communications.

[5] Harish-Chandra, Differential operators on a semisimple Lie algebra, Amer.J.Math. 79 (1957) 87-120 ;

C. Itzykson and J.-B. Zuber, The planar approximation II, J. Math. Phys. 21 (1980) 411-421. 